\title{
Temporal and between-site variation in helminth communities of bank voles (Myodes glareolus) from N.E. Poland. 2. The infracommunity level
}

\author{
J. M. BEHNKE ${ }^{1 *}$, A. BAJER ${ }^{2}$, P. D. HARRIS ${ }^{1}$, L. NEWINGTON $^{1}$, E. PIDGEON $^{1}$, \\ G. ROWLANDS ${ }^{1}$, C. SHERIFF ${ }^{1}$, K. KULIŚ-MALKOWSKA ${ }^{2}$, E. SIŃSKI ${ }^{2}$ \\ F. S. GILBERT ${ }^{1}$ and C. J. BARNARD ${ }^{1}$ \\ ${ }^{1}$ School of Biology, University of Nottingham, University Park, Nottingham NG7 2RD, UK \\ ${ }^{2}$ Department of Parasitology, Institute of Zoology, University of Warsaw, ul. Miecznikowa 1, 02-096 Warszawa, Poland
}

(Received 12 December 2007; revised 27 February and 19 March 2008; accepted 19 March 2008; first published online 2 June 2008)

\author{
SUMMARY
}

The relative importance of temporal and spatial effects was assessed in helminth communities of bank voles (Myodes glareolus) in 3 woodland sites in N.E. Poland in the late summers of 1999 and 2002. Among common species the rank order of sites in relation to prevalence and abundance of infection was maintained between surveys. Site effects accounted for most of the deviance (in statistical models), and time was less important, so the exact location from which voles were sampled was of critical importance. The only exception was Syphacia petrusewiczi. In contrast, for derived measures such as species richness and diversity, most deviance was accounted for by host age, and the interaction between site and year was significant, implying that rank order of sites changed between years. Temporal effects on derived measures were generated primarily by a combination of relatively small changes in prevalence and abundance of the common, rather than the rare, species between the years of the study. In the medium-term, therefore, helminth communities of bank voles in N.E. Poland had a stable core, suggesting a substantial strong element of predictability.

Key words: infracommunities, bank voles, Myodes glareolus, Clethrionomys, helminths, nematodes, cestodes, site-specific parasite variation.

\section{INTRODUCTION}

Helminth communities in hosts can be viewed at various levels of organization, ranging from the entire helminth fauna of a host taxon throughout its entire geographical range, through regional helminth faunas and component communities, to the infracommunities infecting individual hosts (Poulin, 1997; Behnke et al. 2008). Each level experiences different evolutionary and ecological pressures, but the degree to which these communities vary spatially and temporally has not been assessed adequately, even in such well-studied hosts as rodents. This may be surprising given the wealth of studies of rodent helminth communities, but few address this fundamental subject in sufficient detail (Kisielewska, $1970 a$; Haukisalmi et al. 1987; Montgomery and Montgomery, 1989,1990; Tenora and Stanek, 1995). The issue of what a community is, and what structure, stability and predictability it might have, is crucial to establish before launching into complex theoretical treatments of communities.

* Corresponding author: School of Biology, University of Nottingham, University Park, Nottingham NG7 2RD, UK. Tel: 0044 (0) 115951 3208. E-mail: jerzy.behnke@ nottingham.ac.uk
In the preceding paper (Behnke et al. 2008) we found, counter to our expectations, that indices of regional and component community structure for helminths in the bank vole Myodes glareolus were remarkably dynamic temporally. This was surprising because we had expected greater stability at the higher levels of organization. Our analysis showed that the rank order of indices reflecting component community structure in 3 study sites in N.E. Poland was not maintained over a 3-year interval. We attributed this unexpected dynamism primarily to 3 helminths (Syphacia petrusewiczi, Mesocestoides lineatus and Cladotaenia globifera) each of which was rare, but which could achieve extremely high worm burdens in individual hosts, therefore contributing disproportionally to indices of parasite diversity.

We might expect more variation within relatively short-lived infracommunities, which change throughout the host's lifetime (Poulin, 1997). These are influenced by intrinsic factors such as host age because, generally, helminths are long-lived and the overall probability of the host being infected increases with age. Age effects have been quantified and replicated in many studies (Kisielewska, 1971; Montgomery and Montgomery, 1989; Behnke et al. 1999, 2004; Gregory et al. 1992; Bajer et al. 2005) and should now be included routinely when 
examining a sufficiently large sample of hosts spanning different age cohorts. Differences attributable to host sex are also documented, but are less consistent and not always replicable for specific host-parasite combinations (Kisielewska, 1970b; Poulin, 1996; Behnke et al. 2001; Ferrari et al. 2004; Eira et al. 2006).

Extrinsic factors such as season, year of study, site, environment etc. have also been assessed to lesser or greater extents (Lewis, 1968; Langley and Fairley, 1982; Haukisalmi et al. 1988; Montgomery and Montgomery, 1989; Abu-Madi et al. 2000). However, seldom has the contribution of any specific quantified factor been evaluated in a manner that then allows its importance to be clearly established relative to other factors. Which factors are relatively the most important, and which the most predictable, in terms of their influence on infracommunity structures? Do summary measures of infracommunity structure show stability with time in different host populations and, if so, are the differences maintained between surveys? How do common versus rare species contribute to infracommunity structure? Our earlier work showed some temporal stability in helminth infracommunities in bank voles from one of our study sites (Urwitałt; see Bajer et al. 2005) leading us to predict that, with age and sex effects taken into consideration, differences between sites would be a major factor in explaining variation in our measures of infracommunity structure, whereas time would be relatively less important. In this paper we test this idea.

\section{MATERIALS AND METHODS}

\section{Study sites}

The study sites were described comprehensively by Behnke et al. (2001). They are located in Mazury in the north eastern corner of Poland, in the vicinity of Jezioro (Lake) Śniardwy and the towns of Mikołajki, Ryn and Pisz. Site 1 is referred to as Urwitałt (N $53^{\circ} 48 \cdot 153$, EO $21^{\circ} 39 \cdot 784$ ), Site 2 as Talty $\left(\mathrm{N} 53^{\circ} 53 \cdot 644\right.$, EO $\left.21^{\circ} 33.049\right)$ and Site 3 as Pilchy (N $53^{\circ} 42 \cdot 228$, EO $\left.21^{\circ} 48 \cdot 499\right)$ after nearby settlements.

\section{Collection of voles}

In this paper we refer to bank voles as Myodes glareolus following the recent recommendations that Myodes rather than Clethrionomys is the valid name for this genus of rodents (Carleton et al. 2003; Wilson and Reeder, 2005). Methods used for trapping rodents, and for sampling and processing trapped animals have all been fully described by Behnke et al. (2001). Age categories were established as described by Behnke et al. (2001). The breakdown of voles sampled by year, site and age is given in the accompanying paper (Behnke et al. 2008).

\section{Measures of community structure}

Infracommunity structure was assessed following Kennedy and Hartvigsen (2000) by mean number of helminth species per vole, maximum number of helminths and species density distribution across the sample, mean number of helminth individuals per vole, mean Brillouin's Index of Diversity (ID) per vole (infected and uninfected), maximum Brillouin's ID, mean abundance and prevalence of individual species. Brillouin's index of diversity is considered to be the most appropriate for fully censussed communities such as those of the alimentary tract (Kennedy et al. 1986; but see also Washington, 1984). In some analyses, we also excluded the rare species whose overall prevalence was less than $10 \%$, in order to specifically evaluate their relative contribution to infracommunity structure.

\section{Statistical analysis}

The statistical approach adopted has been documented comprehensively in our earlier publications (Behnke et al. 2001; Bajer et al. 2005). We used general linear models (GLM) with Poisson errors (for species richness) or normal errors (for Brillouin's index of diversity) implemented in $\mathrm{R}$ version 2.2.1 (R Core Development Team). Full factorial models that converged satisfactorily were simplified using the STEP procedure and tested for significance using deletion of terms beginning with the highest order interaction by comparing models with or without that interaction. Changes in deviance were interpreted with $\mathrm{Chi}^{2}\left(\chi^{2}\right)$ (Poisson errors), or $F$ (Gaussian errors) tests respectively. Minimum sufficient models were then fitted (all significant interactions and main effects + any main effects that featured in interactions) and the process was repeated to obtain values for changes in deviance, test statistics and probabilities. The percentage of deviance accounted for by each significant main effect or interaction was calculated as recommended by $\mathrm{Xu}$ (2003) using the equation:

percentage deviance explained (by main effect of

factor or interactions between factors)

$$
=1-\left(\left[1-\left\{1-V_{1} / V_{\mathrm{o}}\right\}\right] /\left[1-\left\{1-V_{2} / V_{\mathrm{o}}\right\}\right]\right)
$$

where $V_{\mathrm{o}}$ is the deviance of the null model with no factors or interactions fitted, $V_{1}$ is the deviance of the minimum sufficient model with factor or interaction under evaluation fitted and $V_{2}$ is the deviance of the minimum sufficient model without the factor or interaction under evaluation.

Species density distributions, representing the frequency distributions of infracommunity species richness, were tested for goodness of fit to different distributions as explained by Behnke et al. (2001). 
All distributions were tested for goodness of fit by $\chi^{2}$.

Prevalence data (percentage of animals infected) are shown with $95 \%$ confidence limits, calculated as described by Rohlf and Sokal (1995) employing bespoke software and analysed as described previously (Behnke et al. 2001, 2008) employing maximum likelihood on log linear analysis of contingency tables using the software package SPSS (Version 12.0.1.).

Summary figures for parasite abundance are expressed as arithmetic means \pm s.E.M. These means reflect the abundance of infection as defined by Margolis et al. (1982) and Bush et al. (1997) and include all subjects within the specified group, infected and not infected, for which relevant data were available.

The degree of aggregation in the data was calculated by the index of discrepancy $(D)$ as described by Poulin (1993) and the index of dispersion ( $I$, variance to mean ratio). Frequency distributions of individual taxa were also tested for goodness of fit to negative binomial, positive binomial and Poisson models by $\chi^{2}$ as described by Elliott (1977) and the negative binomial exponent $k$ is given as appropriate.

Abundance data were analysed by fitting GLMs with negative binomial errors, using the glm.nb routine in the MASS library of R (Venables and Ripley, 1997) and, if they converged, simplified as described above and the likelihood ratio statistic $(L R)$ is given. When models in $\mathrm{R}$ with negative binomial errors did not converge, we first explored simpler models. In the case of $H$. mixtum we examined a model with just main effects, and since host sex was not significant we removed this and proceeded further by fitting a full factorial model with all three remaining factors. For analysis of A. tetraptera, models with sex and age would not converge, so the preliminary analysis was conducted fitting a model with just site and year plus their interaction. In the case of $S$. petrusewiczi, no models with interactions would converge. In both cases, $A$. tetraptera and S. petrusewiczi, we used a Box-Cox transformation to normalize the data and fitted models with Gaussian error structures. For all models with negative binomial errors we give the $L R$ statistic and for those with Gaussian errors $F$ is provided.

When the assumptions of parametric tests were not satisfied, the non-parametric Mann-Whitney U-test was used for 2 group comparisons, the 1 -way Kruskal-Wallis ANOVA when more than 2 groups were involved.

We used canonical discriminant function analysis (CDF) in SPSS as an additional approach to evaluating the relative importance of the influence of site and year on parasite burdens. Quantitative parasite data for each of the 13 species of helminths were first standardized (by $\log _{\mathrm{e}}(\mathrm{x}+1)$ transformation of individual worm burdens for each species, then subtraction of mean $\log _{e}$ value for each species and division by the standard deviation) before analysis.

\section{RESULTS}

\section{Mean species richness}

The overall mean number of species harboured per host (all voles combined) was $1 \cdot 48 \pm 0.058$ (variance to mean ratio $=0 \cdot 80)$. There was no significant effect of year (Table 1; Minimum sufficient ANOVA with Poisson errors, main effect of year, $\chi^{2}{ }_{1}=0 \cdot 06$, $P=\mathrm{NS})$, the values being very close in both years (1.44 in 1999 and 1.50 in 2002). However, there was a significant difference between sites and this varied between the two years (Table 1, 2-way interaction, year $*$ site, $\chi_{2}^{2}=16 \cdot 6, P=0 \cdot 0002$ and main effect of site, $\left.\chi_{2}^{2}=9 \cdot 4, \quad P=0.009\right)$. Mean species richness increased at Urwitalt by $25 \cdot 8 \%$ and was the highest of the three sites in 2002. It similarly increased at Talty by $25 \cdot 0 \%$, but fell at Pilchy by $32 \cdot 7 \%$, making this site the poorest in terms of mean species richness in 2002. Thus, the interaction between year and site explained more of the deviance than either factor alone (Table 2).

In contrast to the role played by extrinsic factors, here the intrinsic factor host age was considerably more important in explaining deviance (Table 2), mean species richness increasing with age (Fig. 1A; main effect of age, $\left.\chi_{2}^{2}=43 \cdot 1, P<0 \cdot 0001\right)$. Moreover, there was an indication that the rate of increase with age varied significantly between years (borderline significance for 2 -way interaction year $*$ age $\chi_{2}^{2}=5 \cdot 7$, $P=0 \cdot 058$ ), being relatively slower and spanning a lower range in 1999 compared with 2002 (Fig. 1A).

Mean species richness did not differ significantly between the sexes $($ males $=1 \cdot 43 \pm 0 \cdot 079$, females $=$ $1 \cdot 52 \pm 0 \cdot 084)$.

When all the rare species (those with an overall prevalence of less than $10 \%$, including S. petrusewiczi, T. muris, A. annulosa and all cestodes except $C$. henttoneni), were excluded from these analyses, the values changed only marginally. The largest change to mean species richness was at Urwitalt where exclusion of the rare species resulted in a fall of $18.4 \%$ in 2002 (Table 1). The largest change between years was at Talty, now a $46 \cdot 6 \%$ increase (rather than $24 \cdot 6 \%$ increase when all species were included). Overall mean species richness fell by $13 \cdot 5 \%$ to $1 \cdot 28$.

\section{Infracommunity diversity}

The maximum number of helminth species per vole ranged from 2 in 1999 in Talty to 5 in Urwitalt in 2002 (Table 1), and the exclusion of rare species made little difference to these values.

The mean number of helminths (all species combined) harboured per vole underwent a major 
Table 1. Measures of infracommunity structure by site and year of study, and based on all species of helminths recovered or after exclusion of the rare species

\begin{tabular}{|c|c|c|c|c|c|}
\hline & \multirow[b]{2}{*}{ Year } & \multicolumn{4}{|l|}{ Site } \\
\hline & & Urwitalt & Tałty & Pilchy & Combined \\
\hline $\begin{array}{l}\text { Mean no. of species } \pm \text { s.E.M. } \\
\text { (All } 13 \text { species) }\end{array}$ & $\begin{array}{l}1999 \\
2002 \\
\text { Combined }\end{array}$ & $\begin{array}{l}1 \cdot 53 \pm 0 \cdot 129 \\
2 \cdot 06 \pm 0 \cdot 161 \\
\mathbf{1} \cdot \mathbf{8 7} \pm \mathbf{0} \cdot \mathbf{1 1 5}\end{array}$ & $\begin{array}{l}0 \cdot 98 \pm 0 \cdot 123 \\
1 \cdot 30 \pm 0 \cdot 120 \\
\mathbf{1} \cdot \mathbf{1 8} \pm \mathbf{0} \cdot \mathbf{0 8 9}\end{array}$ & $\begin{array}{l}1 \cdot 71 \pm 0 \cdot 121 \\
1 \cdot 15 \pm 0 \cdot 113 \\
\mathbf{1} \cdot \mathbf{3 9} \pm \mathbf{0} \cdot \mathbf{0 8 6}\end{array}$ & $\begin{array}{l}1 \cdot 44 \pm 0 \cdot 076 \\
1 \cdot 50 \pm 0 \cdot 081 \\
\mathbf{1} \cdot \mathbf{4 8} \pm \mathbf{0} \cdot \mathbf{0 5 8}\end{array}$ \\
\hline $\begin{array}{l}\text { Mean no. of species } \pm \text { s.E.M. } \\
\text { Without rare species* }\end{array}$ & $\begin{array}{l}1999 \\
2002 \\
\text { Combined }\end{array}$ & $\begin{array}{l}1 \cdot 35 \pm 0 \cdot 105 \\
1 \cdot 68 \pm 0 \cdot 126 \\
\mathbf{1} \cdot \mathbf{5 6} \pm \mathbf{0} \cdot \mathbf{0 9 0}\end{array}$ & $\begin{array}{l}0 \cdot 63 \pm 0 \cdot 115 \\
1 \cdot 18 \pm 0 \cdot 106 \\
\mathbf{0} \cdot \mathbf{9 8 2} \pm \mathbf{0} \cdot \mathbf{0 8 3}\end{array}$ & $\begin{array}{l}1 \cdot 57 \pm 0 \cdot 105 \\
1 \cdot 10 \pm 0 \cdot 111 \\
\mathbf{1} \cdot \mathbf{3 0} \pm \mathbf{0 \cdot 0 8 0}\end{array}$ & $\begin{array}{l}1 \cdot 23 \pm 0 \cdot 071 \\
1 \cdot 32 \pm 0 \cdot 068 \\
\mathbf{1} \cdot \mathbf{2 8} \pm \mathbf{0} \cdot \mathbf{0 5 0}\end{array}$ \\
\hline $\begin{array}{l}\text { Maximum number of species } \\
\text { All species (without rare species*) }\end{array}$ & $\begin{array}{l}1999 \\
2002 \\
\text { Combined }\end{array}$ & $\begin{array}{l}(3) \\
(4) \\
(4)\end{array}$ & $\begin{array}{ll}2 & (2) \\
4 & (3) \\
4 & (3)\end{array}$ & $\begin{array}{ll}4 & (3) \\
4 & (4) \\
4 & \text { (4) }\end{array}$ & $\begin{array}{ll}4 & (3) \\
5 & (4) \\
\mathbf{5} & \mathbf{( 4 )}\end{array}$ \\
\hline $\begin{array}{l}\text { Mean no. of helminths } \pm \text { s.E.M. } \\
\text { All species }\end{array}$ & $\begin{array}{l}1999 \\
2002 \\
\text { Combined }\end{array}$ & $\begin{array}{l}109 \cdot 9 \pm 100 \cdot 52 \\
19 \cdot 9 \pm 7 \cdot 14 \\
\mathbf{5 2} \cdot \mathbf{1} \pm \mathbf{3 6} \cdot \mathbf{1 3}\end{array}$ & $\begin{array}{l}126 \cdot 9 \pm 71 \cdot 96 \\
10 \cdot 3 \pm 5 \cdot 49 \\
\mathbf{5 2 \cdot 3} \pm \mathbf{2 6} \cdot \mathbf{4 4}\end{array}$ & $\begin{array}{l}16 \cdot 0 \pm 4 \cdot 03 \\
10 \cdot 6 \pm 3 \cdot 87 \\
\mathbf{1 3} \cdot 0 \pm 2 \cdot 80\end{array}$ & 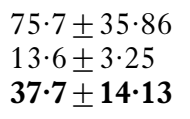 \\
\hline $\begin{array}{l}\text { Mean no. of helminths } \pm \text { s.E.M. } \\
\text { Without rare species }\end{array}$ & $\begin{array}{l}1999 \\
2002 \\
\text { Combined }\end{array}$ & $\begin{array}{l}6 \cdot 13 \pm 0 \cdot 655 \\
6 \cdot 63 \pm 1 \cdot 418 \\
\mathbf{6} \cdot \mathbf{4 5} \pm \mathbf{0 \cdot 9 3 9}\end{array}$ & $\begin{array}{l}9 \cdot 22 \pm 5 \cdot 994 \\
4 \cdot 70 \pm 0 \cdot 860 \\
\mathbf{6} \cdot \mathbf{3 2} \pm \mathbf{2} \cdot \mathbf{2 1 8}\end{array}$ & $\begin{array}{l}15 \cdot 81 \pm 3 \cdot 991 \\
6 \cdot 78 \pm 1 \cdot 914 \\
\mathbf{1 0 \cdot 7 5} \pm \mathbf{2 \cdot 0 8 4}\end{array}$ & $\begin{array}{l}11 \cdot 08 \pm 2 \cdot 445 \\
6 \cdot 04 \pm 0 \cdot 846 \\
\mathbf{7 \cdot 9 9} \pm \mathbf{1 \cdot 0 8 7}\end{array}$ \\
\hline $\begin{array}{l}\text { Mean Brillouin's index } \pm \text { s.E.M. } \\
\text { All species }\end{array}$ & $\begin{array}{l}1999 \\
2002 \\
\text { Combined }\end{array}$ & $\begin{array}{l}0 \cdot 15 \pm 0 \cdot 032 \\
0 \cdot 34 \pm 0 \cdot 038 \\
\mathbf{0} \cdot \mathbf{2 7} \pm \mathbf{0} \cdot \mathbf{0 2 8}\end{array}$ & $\begin{array}{l}0 \cdot 06 \pm 0 \cdot 020 \\
0 \cdot 16 \pm 0 \cdot 028 \\
\mathbf{0} \cdot \mathbf{1 3} \pm \mathbf{0} \cdot \mathbf{0 2 0}\end{array}$ & $\begin{array}{l}0 \cdot 25 \pm 0 \cdot 034 \\
0 \cdot 15 \pm 0 \cdot 031 \\
\mathbf{0 \cdot 1 9} \pm \mathbf{0 \cdot 0 2 3}\end{array}$ & $\begin{array}{l}0 \cdot 17 \pm 0 \cdot 019 \\
0 \cdot 22 \pm 0 \cdot 019 \\
\mathbf{0} \cdot \mathbf{2 0} \pm \mathbf{0} \cdot \mathbf{0 1 4}\end{array}$ \\
\hline $\begin{array}{l}\text { Mean Brillouin's index } \pm \text { s.E.M. } \\
\text { Without rare species* }\end{array}$ & $\begin{array}{l}1999 \\
2002 \\
\text { Combined }\end{array}$ & $\begin{array}{l}0 \cdot 12 \pm 0 \cdot 027 \\
0 \cdot 26 \pm 0 \cdot 033 \\
\mathbf{0} \cdot \mathbf{2 1} \pm \mathbf{0} \cdot \mathbf{0 2 4}\end{array}$ & $\begin{array}{l}0 \cdot 04 \pm 0 \cdot 015 \\
0 \cdot 14 \pm 0 \cdot 026 \\
\mathbf{0} \cdot \mathbf{1 0} \pm \mathbf{0} \cdot \mathbf{0 1 8}\end{array}$ & $\begin{array}{l}0 \cdot 23 \pm 0 \cdot 032 \\
0 \cdot 14 \pm 0 \cdot 030 \\
\mathbf{0} \cdot \mathbf{1 8} \pm \mathbf{0} \cdot \mathbf{0 2 2}\end{array}$ & $\begin{array}{l}0 \cdot 14 \pm 0 \cdot 017 \\
0 \cdot 18 \pm 0 \cdot 017 \\
\mathbf{0} \cdot \mathbf{1 7} \pm \mathbf{0} \cdot \mathbf{0 1 3}\end{array}$ \\
\hline Maximum Brillouin's index & $\begin{array}{l}1999 \\
2002 \\
\text { Combined }\end{array}$ & $\begin{array}{l}0 \cdot 653 \\
1 \cdot 123 \\
\mathbf{1} \cdot 123\end{array}$ & $\begin{array}{l}0 \cdot 461 \\
0 \cdot 819 \\
\mathbf{0} \cdot \mathbf{8 1 9}\end{array}$ & $\begin{array}{l}0 \cdot 968 \\
1 \cdot 105 \\
\mathbf{1} \cdot \mathbf{1 0 5}\end{array}$ & $\begin{array}{l}0 \cdot 968 \\
1 \cdot 123 \\
\mathbf{1} \cdot \mathbf{1 2 3}\end{array}$ \\
\hline
\end{tabular}

* For these analyses we excluded all species for which prevalence was less than $10 \%$. Therefore the analysis was based on 5 species which were $H$. mixtum, $H$. glareoli, A. tetraptera, $M$. muris and $C$. henttoneni.

change, falling overall from a mean of $75 \cdot 7$ to just 13.6 in 2002 (Table 1 and $82 \%$ reduction; minimum sufficient model with negative binomial errors, main effect of year, $\left.L R_{1}=67 \cdot 9, P<0 \cdot 001\right)$. This year effect accounted for more of the deviance than any of the other terms in the model (Table 2) and was particularly evident in Talty (Table $5,91 \cdot 9 \%$ reduction) and Urwitałt (81.9\%), but less so in Pilchy (34.1\%). However, there was also a significant 2 -way interaction with site (site $*$ year, $L R_{2}=18 \cdot 2, P=0 \cdot 0001$ ) and the main effect of site was significant $\left(L R_{2}=7 \cdot 3\right.$, $P=0.025)$, but both accounted for less explained deviance than the year effect (Table 2).

The analysis also revealed a highly significant main effect of age $\left(L R_{1}=35 \cdot 8, P<0 \cdot 001\right)$. This was evident in both years (Fig. 1B) but the differences between age classes were greater in 2002 compared with 1999, although not significantly so (no significant 2 -way interaction between year and age). The model also had a significant 2 -way interaction between site and age $\left(L R_{4}=28 \cdot 5, P<0 \cdot 0001\right)$ but no clear pattern emerged when this was scrutinized carefully (not illustrated).

When the rare species were removed from the analysis, the values for the mean number of helminths harboured dropped enormously at both Urwitalt and
Tałty but less so at Pilchy, and this was mainly accounted for by the removal from the dataset of $S$. petrusewiczi. Without the rare species, some of which can generate huge numbers of individuals when present, the values were more stable across sites and between years. In both years the mean number of helminths was highest at Pilchy but because of a reduction at Tałty between 1999 and 2002, Tałty fell from intermediate position to bottom between the surveys. In contrast note the stable values at Urwitalt (Table 1).

Analysis of Brillouin's index of diversity (ID) in $\mathrm{R}$ (with Gaussian errors) yielded a model indicating that age explained the greatest proportion of deviance (Table 2; main effect of age, $F_{2,352}=22 \cdot 4$, $P<0 \cdot 0001)$, but that the effect of age varied significantly between years. As Fig. 1D shows the age effect was clearly evident in both years of the study, although the increase in Brillouin's ID was steeper with age in 2002 compared with 1999 (age*year, $\left.F_{2,350}=5 \cdot 4, P=0 \cdot 005\right)$. The age effect was similar in all three sites (Fig. 1C).

Overall Brillouin's ID was very similar in 1999 and 2002 (Table $1,0 \cdot 17$ and $0 \cdot 22$, respectively; for main effect of year $\left.F_{1,352}=1 \cdot 4, P=0 \cdot 2\right)$, but there were differences between sites (main effect of site, 
Table 2. Percentage of variation in data (deviance) explained by extrinsic and intrinsic factors affecting the measures of infracommunity structure and diversity, and the abundance of helminths

(In each case the output from the most parsimonious and appropriate minimum sufficient model is given. Thus, only the significant main effects and interactions, and non-significant main effects if a component of one of the interactions, have been included. Models for total helminth burden and individual species are models with negative binomial error structures unless stated otherwise below. For further details of the statistical models, see the text. Note that some 2-way and 3-way interactions and the 4-way interaction are not given because these were not significant.)

\begin{tabular}{|c|c|c|c|c|c|c|c|c|c|c|}
\hline \multirow{2}{*}{$\begin{array}{l}\text { Source } \\
\text { of } \\
\text { variation }\end{array}$} & \multicolumn{10}{|c|}{ Percentage of deviance explained } \\
\hline & $\operatorname{MSR}^{1}$ & $\mathrm{BID}^{2}$ & TotHelms $^{3}$ & H. mixtum & H. glareoli & M. muris & C. henttoneni & A.te & raptera ${ }^{4}$ & S. petrusewiczi $i^{4}$ \\
\hline Site & $3 \cdot 22$ & $3 \cdot 03$ & $0 \cdot 29$ & $18 \cdot 89$ & $21 \cdot 52$ & $7 \cdot 16$ & $9 \cdot 42$ & $1 \cdot 09$ & $(17 \cdot 18)$ & $3 \cdot 92$ \\
\hline Year & $0 \cdot 21$ & $0 \cdot 39$ & $2 \cdot 59$ & $2 \cdot 61$ & $5 \cdot 082$ & - & $4 \cdot 45$ & $0 \cdot 07$ & $(2 \cdot 59)$ & $5 \cdot 10$ \\
\hline Age & $13 \cdot 28$ & $11 \cdot 30$ & $1 \cdot 38$ & $2 \cdot 40$ & - & $4 \cdot 49$ & $1 \cdot 47$ & nd & $(3 \cdot 90)$ & - \\
\hline Sex & - & - & - & - & - & $2 \cdot 11$ & $0 \cdot 01$ & nd & - & $0 \cdot 05$ \\
\hline Site $*$ age & - & - & $1 \cdot 12$ & - & - & $2 \cdot 31$ & $2 \cdot 03$ & nd & - & - \\
\hline Site $*$ year & $6 \cdot 08$ & $7 \cdot 28$ & $0 \cdot 72$ & $1 \cdot 35$ & - & $2 \cdot 14$ & - & $2 \cdot 42$ & $(4 \cdot 43)$ & $6 \cdot 13$ \\
\hline Site $* \operatorname{sex}$ & - & - & - & - & - & - & - & - & - & $2 \cdot 27$ \\
\hline Year * age & $2 \cdot 18$ & $3 \cdot 02$ & - & $2 \cdot 17$ & - & $1 \cdot 30$ & - & - & - & - \\
\hline $\begin{array}{l}\text { Site } * \text { age } * \\
\text { sex }\end{array}$ & - & - & - & - & - & - & $6 \cdot 16$ & nd & - & - \\
\hline
\end{tabular}

Change in relative ranking between sites

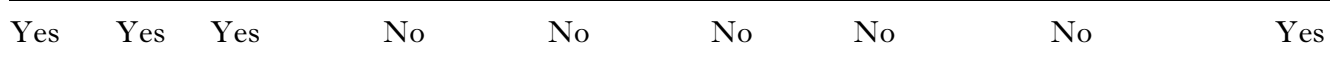

1. Mean species richness (statistical model based on Poisson errors).

2. Brillouin's index of diversity (statistical model based on Normal errors).

3. Total helminth burden.

4. GLM models for these species were problematic and percentage deviance could not be reliably estimated in the context of all factors.

For A.tetraptera we give the output from two models: the first with negative binomial errors fitting only the main effects of site and year and their interaction; the second in parenthesis for a minimum sufficient model on BoxCox transformed data, with normal error structure.

For $S$. petrusewiczi we give the output for a minimum sufficient model on BoxCox transformed data, with normal error structure.

$\left.F_{2,352}=5 \cdot 5, P=0 \cdot 004\right)$ and these changed between the years of the study (2-way interaction site*year, $\left.F_{2,350}=13 \cdot 7, P<0 \cdot 0001\right)$. In 1999 the lowest index was at Talty and the highest at Pilchy, but by 2002 the index had increased at both Urwitalt and Tałty and dropped in Pilchy.

There was no overall difference in Brillouin's ID between the sexes $($ males $=0 \cdot 19 \pm 0 \cdot 019$, females $=$ $0 \cdot 20 \pm 0 \cdot 021)$.

Exclusion of the rare species (Table 1) made very little difference to the mean values of Brillouin's ID recorded for the three sites and in both years. Overall (sites and years combined) it fell by $15 \%$ from $0 \cdot 2$ to $0 \cdot 17$, and the range of change was from $5 \cdot 3 \%$ to $23 \cdot 5 \%$. As with mean species richness the biggest change was recorded at Urwitałt.

\section{Species density distributions}

In the combined data-set and in each year, with sites combined (Fig. 2B), the modal class was just 1 species of helminth $(35 \cdot 2-40 \cdot 3 \%$ of voles $)$. This was also the case for 4 of the 6 subsets of data (Fig. 2A), but in 1999 at Pilchy the modal class was 2 species of helminths $(39 \cdot 7 \%)$ and in 2002 at Urwitalt it was 3 species $(26 \cdot 4 \%)$. Four-species infections were rare in 1999 (only 1 vole from Pilchy), but were present in all three sites in 2002. The difference between the distributions in 1999 and 2002 had borderline significance (with sites combined; $\chi^{2}{ }_{5}=10 \cdot 4, P=0 \cdot 06$ ). In 2002 there were relatively more (in percentage terms) uninfected voles, fewer with 1 and 2 species of helminths but more with 3, 4 and 5 helminth species. The combined data-set (Fig. 2C) did not differ significantly from the null model for interactions of parasite species in an assemblage (Janovy et al. 1995 ; $\left.\chi_{4}^{2}=2 \cdot 11, P=0 \cdot 83\right)$.

\section{Prevalence of species and higher taxa}

Table 3 shows that by 2002 , overall prevalence of helminths had dropped by $7 \cdot 1 \%$ (from 85.6 to $78.5 \%$ ), but the extent of the change between the two surveys varied between sites (year*site $*$ presence/ absence of helminths, $\left.\chi_{2}^{2}=11 \cdot 1, P=0 \cdot 004\right)$. Thus, whereas at Urwitalt and Pilchy prevalence of helminths dropped between 1999 and 2002 (9.4 and $20 \cdot 1 \%$, respectively), at Talty it actually increased by $12 \cdot 3 \%$. The prevalence of helminths also increased significantly with increasing host age (Fig. 3A; 

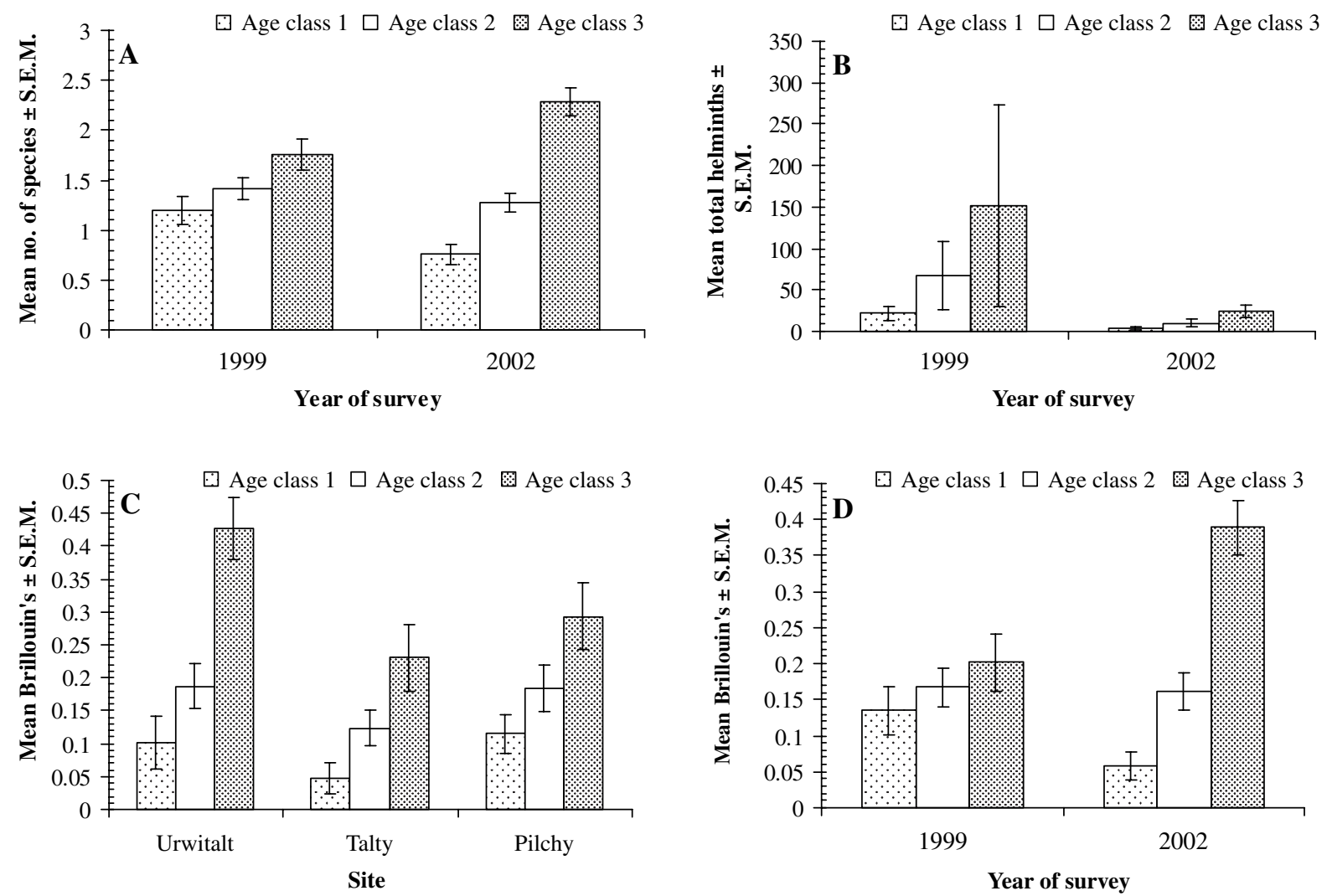

Fig. 1. Age-related variation in measures of infracommunity structure. (A) Variation in mean no. of helminth species between the two years of the study. (B) Variation in mean total helminth burden with year of survey. (C) Variation in Brillouin's index of diversity by site (both years pooled). (D) Variation in Brillouin's index by year of survey.

age $*$ presence/absence of helminths, $\quad \chi_{2}^{2}=29 \cdot 4$, $P<0 \cdot 0001)$.

The overall reduction in prevalence of total helminths between the two surveys was mainly accounted for by nematodes (an overall reduction of $12.5 \%)$, falling at both Urwitalt and Pilchy but staying virtually identical at Talty (Table 3 ; year* site $*$ presence/absence of nematodes, $\chi_{2}^{2}=8 \cdot 9, P=$ $0 \cdot 012$ ). For nematodes there was also a highly significant increase in prevalence with host age (Fig. 3B ; age $*$ presence/absence of nematodes, $\chi_{2}^{2}=34 \cdot 3, P<$ 0.0001).

The greatest overall percentage change between the years was observed with $H$. glareoli, the second most prevalent species in 1999, with prevalence falling overall from $36 \cdot 0 \%$ in 1999 to $10 \cdot 5 \%$ in 2002 . This drop in prevalence is apparent in Fig. 3C, but as can be seen it was confounded by variation between the sexes and age classes (year*sex $*$ age $*$ presence/ absence of $H$. glareoli, $\left.\chi_{2}^{2}=7 \cdot 9, P=0 \cdot 020\right)$. There was also an independent marked difference between sites (Table 3, site $*$ presence/absence of $H$. glareoli, $\left.\chi_{2}^{2}=123 \cdot 7, P<0 \cdot 0001\right)$, with prevalence being relatively high at Pilchy in both years and comparatively low at the other two sites. So at this level, the site effect was maintained between the two years of the study. However, because essentially this species was largely encountered at just the 1 site, in a second step the analysis was confined to voles from Pilchy, where overall prevalence was $50 \cdot 8 \%$. The difference between years was now highly significant (year* presence/absence of $H$. glareoli, $\chi_{1}^{2}=35 \cdot 5, \quad P<$ $0 \cdot 0001)$ and no other factors came into the equation. Prevalence at Pilchy was marginally higher in females $(52 \cdot 2 \%$ [41.1-63.1]) compared with males $(49 \cdot 3 \%[37 \cdot 8-60 \cdot 7])$ but this was not significant and it fell gradually although not significantly with increasing age (from $53 \cdot 7 \%[37 \cdot 2-69 \cdot 0]$ in age class 1 , $50 \cdot 9 \%[40 \cdot 7-61 \cdot 2]$ in age class 2 to $47 \cdot 4 \%$ [31·0-62.9] in age class 3 voles.

H. mixtum was the most prevalent parasite in 1999 , but not in 2002, when $A$. tetraptera was more common (Table 3 ). The strongest effect on the prevalence of $H$. mixtum was host age, prevalence increasing significantly with increasing age (Fig. 3D; age $*$ presence/absence of $H$. mixtum, $\chi^{2}{ }_{2}=29 \cdot 1, P<$ $0 \cdot 0001)$. Overall the prevalence of $H$. mixtum fell by $7.9 \%$ between the two surveys. The reduction was most marked at Urwitalt $(31 \cdot 1 \%$ reduction), but was also evident at Talty $(9 \cdot 7 \%)$, and this difference between sites and the degree to which prevalence changed at each was significant (Table 3 ; year $*$ site $*$ presence/absence of $H$. mixtum, $\chi_{2}^{2}=6 \cdot 6$, $P=0.0369)$. H. mixtum was not encountered in either 

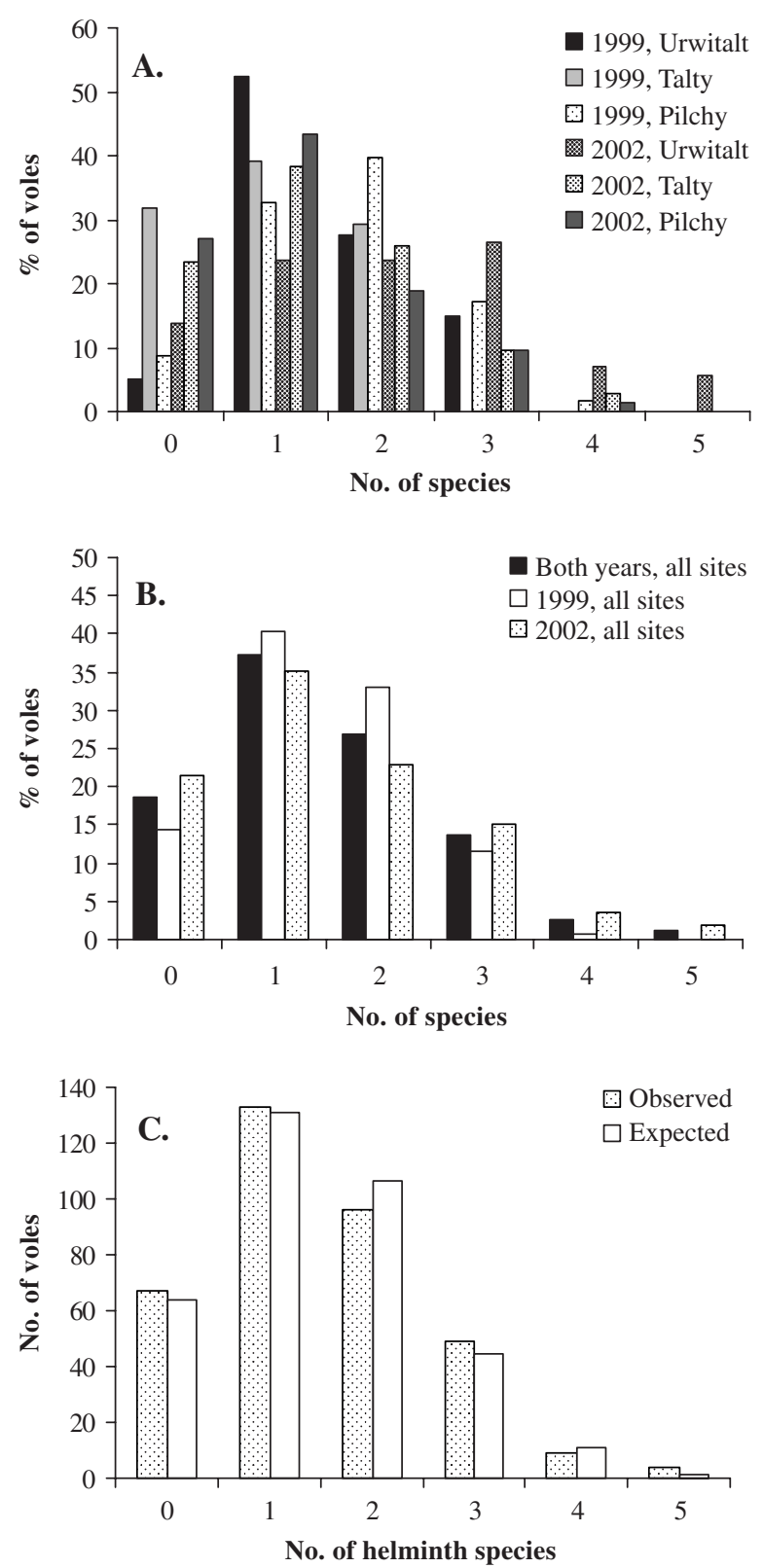

Fig. 2. Species density distributions by site and year of survey. (A) All three sites in both surveys. (B) Data combined by year of survey and both years combined. (C) All observed data combined, and the pattern predicted (expected) by the null model of Janovy et al. (1995).

year at Pilchy. There was no significant difference in prevalence between sexes (males $=36 \cdot 6 \%$ [27 $8-46 \cdot 1]$ and females $=34 \cdot 3 \%[26 \cdot 0-43 \cdot 4]$ ).

In contrast to $H$. mixtum and $H$. glareoli, the prevalence of $A$. tetraptera increased overall by 16.4\% between 1999 and 2002 (Table 3). At Pilchy, which had shown the highest prevalence in 1999, there was little change and it was still the site with the highest prevalence of this species. However, as can be seen from Table 3, at both of the other sites prevalence increased quite markedly (year $*$ site $*$ presence/ absence of $A$. tetraptera, $\left.\chi_{2}^{2}=20 \cdot 1, P<0 \cdot 0001\right)$ but overall the relative ranking of sites was maintained across the two years. There was also a significant increase in prevalence with host age (Fig. 3E, age* presence/absence of $A$. tetraptera, $\chi_{2}^{2}=16 \cdot 1, P=$ $0 \cdot 0003)$, but prevalence was almost identical in both sexes $\quad($ males $=38 \cdot 7 \% \quad[30 \cdot 0-48 \cdot 3]$ and females $=$ $39 \cdot 0 \%[30 \cdot 6-48 \cdot 1])$.

Mastophorus muris was another species that appeared to increase in prevalence, this time at all three sites, although not to the same degree (Table 3). When other factors were taken into account the year effect was not significant. However, there was a highly significant effect of site (Table 3 ; site $*$ presence/absence of $M$. muris, $\left.\chi_{2}^{2}=38 \cdot 1, P<0 \cdot 0001\right)$. The highest prevalence in 1999 was at Pilchy but in 2002 prevalence was higher at Urwitałt, where voles showed a $23 \cdot 3 \%$ increase between the two surveys. At Tałty this species continued to be relatively rare, so between the two surveys there was a reversal of ranking among the two sites where the parasite was common. Prevalence increased significantly with host age, with a particularly steep rise between age classes 2 and 3 (Fig. 3F, age* presence/absence of M. muris, $\left.\chi_{2}^{2}=25 \cdot 7, P<0 \cdot 0001\right)$. In contrast to other species, there was a pronounced sex effect, female voles showing 2.4 times higher prevalence than males (males $=9 \cdot 1 \%[4 \cdot 6-16 \cdot 2]$ and females $=21 \cdot 5 \%$ [14.9-30.0]; sex* presence/absence of $M$. muris, $\left.\chi_{1}^{2}=10 \cdot 9, P=0 \cdot 001\right)$.

The prevalence of $S$. petrusewiczi only varied in relation to site and year of survey (year $*$ site $*$ presence/absence of $S$. petrusewiczi, $\chi_{2}^{2}=8 \cdot 6, P=$ $0 \cdot 013)$. At all three sites prevalence declined between 1999 and 2002 (Table 3), but the change was the greatest at Talty $(30 \cdot 3 \%)$, where in 1999 prevalence of this species had been the highest. There was no significant difference in prevalence between the sexes $($ males $=6 \cdot 5 \%[2 \cdot 9-13 \cdot 0]$ and females $=7 \cdot 5 \%[3 \cdot 6-$ $14 \cdot 2])$.

The remaining nematodes were rare. A. annulosa was only detected in 2002 at Urwitalt and Talty but not at Pilchy and, interestingly was recovered only from 6 female voles of age class 3. Trichuris sp. was only recovered from 1 vole in 1999 from Pilchy.

In contrast to nematodes, the overall prevalence of cestodes increased by $17 \cdot 7 \%$ (larval cestodes $8 \cdot 5 \%$ and adult cestodes $17 \cdot 9 \%$ ) between 1999 and 2002, the increase occurring mainly in voles from Urwitalt and Talty (Table 3). This difference between years and sites was significant but was also confounded by host sex (year $*$ site $*$ sex $*$ presence/absence of all cestodes, $\left.\chi_{2}^{2}=7 \cdot 9, P=0 \cdot 019\right)$, as illustrated in Fig. 4A. The site and sex effect was also complicated by host age (Fig. 4B; site $*$ sex $*$ age $*$ presence/absence of all cestodes, $\left.\chi_{4}^{2}=16 \cdot 5, P=0 \cdot 0024\right)$, an age-dependent increase in cestode infections being clearly apparent among male voles at Urwitalt and Pilchy but not at Talty. Despite the variation between age classes, there was no clear pattern among female voles. 
Table 3. Prevalence ( $\%$ infected) of helminth taxa by site and year

\begin{tabular}{|c|c|c|c|c|c|c|}
\hline \multirow[b]{2}{*}{ Taxon } & \multirow[b]{2}{*}{ Species } & \multirow[b]{2}{*}{ Year } & \multicolumn{3}{|l|}{ Site } & \multirow[b]{2}{*}{ Total } \\
\hline & & & Urwitalt* & Talty* & Pilchy* & \\
\hline \multirow[t]{8}{*}{ Nematodes } & $\begin{array}{l}\text { Heligmosomum } \\
\text { mixtum }\end{array}$ & $\begin{array}{l}1999 \\
2002 \\
\text { combined }\end{array}$ & $\begin{array}{l}95 \cdot 0(83 \cdot 9-99 \cdot 2) \\
63 \cdot 9(52 \cdot 5-74 \cdot 3) \\
\mathbf{7 5} \cdot \mathbf{0}(\mathbf{6 6} \cdot \mathbf{2}-\mathbf{8 2} \cdot \mathbf{2})\end{array}$ & $\begin{array}{l}43 \cdot 9(30 \cdot 5-60 \cdot 4) \\
34 \cdot 2(24 \cdot 8-46 \cdot 5) \\
\mathbf{3 7 \cdot 7}(\mathbf{2 9} \cdot \mathbf{6}-\mathbf{4 7} \cdot \mathbf{3})\end{array}$ & $\begin{array}{l}0(0 \cdot 1-8 \cdot 0) \\
0(0 \cdot 1-6 \cdot 6) \\
\mathbf{0}(\mathbf{0} \cdot \mathbf{1}-\mathbf{4} \cdot \mathbf{5})\end{array}$ & $\begin{array}{l}40 \cdot 3(33 \cdot 0-49 \cdot 3) \\
32 \cdot 4(27 \cdot 0-39 \cdot 6) \\
\mathbf{3 5} \cdot \mathbf{5}(\mathbf{3 1} \cdot \mathbf{3}-\mathbf{4 1} \cdot \mathbf{0})\end{array}$ \\
\hline & $\begin{array}{l}\text { Heligmosomoides } \\
\text { glareoli }\end{array}$ & $\begin{array}{l}1999 \\
2002 \\
\text { combined }\end{array}$ & $\begin{array}{l}7 \cdot 5(2 \cdot 8-21 \cdot 6) \\
1 \cdot 4(0 \cdot 3-8 \cdot 6) \\
\mathbf{3} \cdot \mathbf{6}(\mathbf{1} \cdot \mathbf{5}-\mathbf{9} \cdot \mathbf{4})\end{array}$ & $\begin{array}{l}2 \cdot 4(0 \cdot 3-14 \cdot 0) \\
1 \cdot 4(0 \cdot 3-8 \cdot 5) \\
\mathbf{1} \cdot \mathbf{8}(\mathbf{0} \cdot \mathbf{4}-\mathbf{6} \cdot \mathbf{7})\end{array}$ & $\begin{array}{l}79 \cdot 3(67 \cdot 5-88 \cdot 7) \\
28 \cdot 4(19 \cdot 8-40 \cdot 4) \\
\mathbf{5 0 \cdot 8}(\mathbf{4 2 \cdot 4 - 5 9 \cdot 6 )}\end{array}$ & $\begin{array}{l}36 \cdot 0(28 \cdot 4-44 \cdot 3) \\
10 \cdot 5(7 \cdot 4-15 \cdot 9) \\
\mathbf{2 0} \cdot \mathbf{4}(\mathbf{1 7} \cdot \mathbf{1 - 2 5} \cdot \mathbf{4})\end{array}$ \\
\hline & $\begin{array}{l}\text { Syphacia } \\
\text { petrusewiczi }\end{array}$ & $\begin{array}{l}1999 \\
2002 \\
\text { combined }\end{array}$ & $\begin{array}{l}10 \cdot 0(3 \cdot 6-23 \cdot 5) \\
6 \cdot 9(2 \cdot 9-15 \cdot 4) \\
\mathbf{8} \cdot 0(\mathbf{4} \cdot \mathbf{8}-\mathbf{1 5} \cdot 8)\end{array}$ & $\begin{array}{l}31 \cdot 7(19 \cdot 3-47 \cdot 9) \\
1 \cdot 4(0 \cdot 3-8 \cdot 5) \\
\mathbf{1 2 \cdot 3}(\mathbf{7} \cdot \mathbf{7}-\mathbf{2 0} \cdot \mathbf{3})\end{array}$ & $\begin{array}{l}3 \cdot 4(0 \cdot 9-12 \cdot 6) \\
0(0-6 \cdot 6) \\
\mathbf{1} \cdot \mathbf{5}(\mathbf{0} \cdot \mathbf{5}-\mathbf{6} \cdot \mathbf{2})\end{array}$ & $\begin{array}{l}13 \cdot 7(9 \cdot 1-20 \cdot 7) \\
2 \cdot 7(1 \cdot 4-6 \cdot 2) \\
\mathbf{7} \cdot \mathbf{0}(\mathbf{4} \cdot \mathbf{8}-\mathbf{1 0} \cdot \mathbf{1})\end{array}$ \\
\hline & $\begin{array}{r}\text { Aspiculuris } \\
\text { tetraptera }\end{array}$ & $\begin{array}{l}1999 \\
2002 \\
\text { combined }\end{array}$ & $\begin{array}{l}2 \cdot 5(0 \cdot 3-14 \cdot 3) \\
23 \cdot 6(15 \cdot 3-35 \cdot 2) \\
\mathbf{1 6} \cdot \mathbf{1}(\mathbf{1 1} \cdot \mathbf{0}-\mathbf{2 5} \cdot \mathbf{3})\end{array}$ & $\begin{array}{l}12 \cdot 2(5 \cdot 6-26 \cdot 8) \\
53 \cdot 4(42 \cdot 5-64 \cdot 9) \\
\mathbf{3 8} \cdot \mathbf{6}(\mathbf{3 0} \cdot \mathbf{6}-\mathbf{4 8} \cdot \mathbf{3})\end{array}$ & $\begin{array}{l}58 \cdot 6(46 \cdot 1-71 \cdot 2) \\
58 \cdot 1(47 \cdot 6-69 \cdot 8) \\
\mathbf{5 8} \cdot \mathbf{3}(\mathbf{5 0} \cdot \mathbf{5}-\mathbf{6 7} \cdot \mathbf{2})\end{array}$ & $\begin{array}{l}28 \cdot 8(22 \cdot 1-37 \cdot 1) \\
45 \cdot 2(39 \cdot 4-52 \cdot 6) \\
\mathbf{3 8} \cdot \mathbf{8}(\mathbf{3 4} \cdot \mathbf{2}-\mathbf{4 4} \cdot \mathbf{1})\end{array}$ \\
\hline & $\begin{array}{l}\text { Mastophorus } \\
\text { muris }\end{array}$ & $\begin{array}{l}1999 \\
2002 \\
\text { combined }\end{array}$ & $\begin{array}{l}10 \cdot 0(3 \cdot 6-23 \cdot 5) \\
33 \cdot 3(23 \cdot 9-45 \cdot 5) \\
\mathbf{2 5} \cdot 0(\mathbf{1 7} \cdot \mathbf{8}-\mathbf{3 3} \cdot \mathbf{8})\end{array}$ & $\begin{array}{l}0(0-11 \cdot 9) \\
1 \cdot 4(0 \cdot 3-8 \cdot 5) \\
\mathbf{0} \cdot \mathbf{9}(\mathbf{0} \cdot \mathbf{1}-\mathbf{4} \cdot \mathbf{9})\end{array}$ & $\begin{array}{l}17 \cdot 2(10 \cdot 0-29 \cdot 8) \\
20 \cdot 3(13 \cdot 0-31 \cdot 7) \\
\mathbf{1 8} \cdot \mathbf{9}(\mathbf{1 3} \cdot \mathbf{1}-\mathbf{2 6} \cdot \mathbf{6})\end{array}$ & $\begin{array}{l}10 \cdot 1(6 \cdot 6-17 \cdot 4) \\
18 \cdot 3(14 \cdot 2-24 \cdot 8) \\
\mathbf{1 5} \cdot \mathbf{1}(\mathbf{1 2} \cdot \mathbf{6}-\mathbf{2 0} \cdot \mathbf{1})\end{array}$ \\
\hline & $\begin{array}{l}\text { Aonchotheca } \\
\text { annulosa }\end{array}$ & $\begin{array}{l}1999 \\
2002 \\
\text { combined }\end{array}$ & $\begin{array}{l}0(0-12 \cdot 3) \\
5 \cdot 6(2 \cdot 3-14 \cdot 1) \\
\mathbf{3} \cdot \mathbf{6}(\mathbf{1} \cdot \mathbf{5}-\mathbf{9} \cdot \mathbf{4})\end{array}$ & $\begin{array}{l}0(0-11 \cdot 9) \\
2 \cdot 7(0 \cdot 7-9 \cdot 8) \\
\mathbf{1} \cdot \mathbf{8}(\mathbf{0} \cdot \mathbf{4}-\mathbf{6} \cdot \mathbf{7})\end{array}$ & $\begin{array}{l}0(0-8 \cdot 0) \\
0(0-6 \cdot 6) \\
\mathbf{0}(\mathbf{0}-\mathbf{4} \cdot \mathbf{5})\end{array}$ & $\begin{array}{l}0(0-4 \cdot 4) \\
2 \cdot 7(1 \cdot 4-6 \cdot 2) \\
\mathbf{1} \cdot \mathbf{7}(\mathbf{1} \cdot \mathbf{0}-\mathbf{4} \cdot \mathbf{1})\end{array}$ \\
\hline & Trichuris sp. & $\begin{array}{l}1999 \\
2002 \\
\text { combined }\end{array}$ & $\begin{array}{l}0(0-12 \cdot 3) \\
0(0-6 \cdot 8) \\
\mathbf{0}(\mathbf{0}-\mathbf{5} \cdot \mathbf{0})\end{array}$ & $\begin{array}{l}0(0-11 \cdot 9) \\
0(0-6 \cdot 7) \\
\mathbf{0}(\mathbf{0 - 4} \cdot \mathbf{9})\end{array}$ & $\begin{array}{l}1 \cdot 7(0 \cdot 2-9 \cdot 7) \\
(0-6 \cdot 6) \\
\mathbf{0} \cdot \mathbf{8}(\mathbf{0} \cdot \mathbf{1}-\mathbf{4} \cdot \mathbf{5})\end{array}$ & $\begin{array}{l}0 \cdot 7(0 \cdot 1-4 \cdot 4) \\
0(0-3 \cdot 5) \\
\mathbf{0} \cdot \mathbf{3}(\mathbf{0} \cdot 3-2 \cdot 7)\end{array}$ \\
\hline & All nematodes & $\begin{array}{l}1999 \\
2002 \\
\text { combined }\end{array}$ & $\begin{array}{l}95 \cdot 0(83 \cdot 9-99 \cdot 2) \\
80 \cdot 6(70 \cdot 4-88 \cdot 5) \\
\mathbf{8 5} \cdot \mathbf{7}(\mathbf{7 8} \cdot \mathbf{2}-\mathbf{9 1} \cdot \mathbf{3})\end{array}$ & $\begin{array}{l}68 \cdot 3(53 \cdot 9-81 \cdot 6) \\
68 \cdot 5(57 \cdot 6-78 \cdot 7) \\
\mathbf{6 8} \cdot \mathbf{4}(\mathbf{5 9} \cdot \mathbf{8}-\mathbf{7 7} \cdot \mathbf{0})\end{array}$ & $\begin{array}{l}91 \cdot 4(82 \cdot 2-96 \cdot 9) \\
70 \cdot 3(59 \cdot 6-80 \cdot 2) \\
\mathbf{7 9} \cdot \mathbf{5}(\mathbf{7 2} \cdot \mathbf{4}-\mathbf{8 6} \cdot \mathbf{1})\end{array}$ & $\begin{array}{l}85 \cdot 6(79 \cdot 3-90 \cdot 9) \\
73 \cdot 1(67 \cdot 9-79 \cdot 5) \\
\mathbf{7 7} \cdot \mathbf{9}(\mathbf{7 3} \cdot \mathbf{5}-\mathbf{8 2} \cdot \mathbf{0})\end{array}$ \\
\hline \multirow[t]{10}{*}{ Cestodes } & $\begin{array}{l}\text { Catenotaenia } \\
\text { henttoneni }\end{array}$ & $\begin{array}{l}1999 \\
2002 \\
\text { combined }\end{array}$ & $\begin{array}{l}20 \cdot 0(10 \cdot 1-35 \cdot 5) \\
45 \cdot 8(35 \cdot 0-57 \cdot 6) \\
\mathbf{3 6} \cdot \mathbf{6}(\mathbf{2 8} \cdot \mathbf{5}-\mathbf{4 6} \cdot \mathbf{3})\end{array}$ & $\begin{array}{l}\mathbf{4} \cdot 9(1 \cdot 2-17 \cdot 2) \\
27 \cdot 4(18 \cdot 7-39 \cdot 2) \\
\mathbf{1 9} \cdot \mathbf{3}(\mathbf{1 3} \cdot \mathbf{4}-\mathbf{2 8 \cdot 3})\end{array}$ & $\begin{array}{l}1 \cdot 7(0 \cdot 2-9 \cdot 7) \\
2 \cdot 7(0 \cdot 7-9 \cdot 7) \\
\mathbf{2} \cdot \mathbf{3}(\mathbf{1} \cdot \mathbf{1}-\mathbf{7} \cdot \mathbf{4})\end{array}$ & $\begin{array}{l}7 \cdot 9(4 \cdot 4-13 \cdot 8) \\
25 \cdot 1(20 \cdot 5-32 \cdot 1) \\
\mathbf{1 8} \cdot \mathbf{4}(\mathbf{1 5} \cdot \mathbf{3}-\mathbf{2 3} \cdot \mathbf{3})\end{array}$ \\
\hline & $\begin{array}{l}\text { Paranoplocephala } \\
\text { omphalodes }\end{array}$ & $\begin{array}{l}1999 \\
2002 \\
\text { combined }\end{array}$ & $\begin{array}{l}2 \cdot 5(0 \cdot 3-14 \cdot 3) \\
2 \cdot 8(0 \cdot 7-9 \cdot 8) \\
2 \cdot 7(0 \cdot 9-7 \cdot 9)\end{array}$ & $\begin{array}{l}0(0 \cdot 1-11 \cdot 9) \\
1 \cdot 4(0 \cdot 3-8 \cdot 5) \\
\mathbf{0} \cdot \mathbf{9}(\mathbf{0} \cdot \mathbf{1}-\mathbf{4} \cdot \mathbf{9})\end{array}$ & $\begin{array}{l}0(0 \cdot 1-8 \cdot 0) \\
0(0 \cdot 1-6 \cdot 6) \\
\mathbf{0}(\mathbf{0} \cdot \mathbf{1}-\mathbf{4} \cdot \mathbf{5})\end{array}$ & $\begin{array}{l}0 \cdot 7(0 \cdot 1-4 \cdot 4) \\
1 \cdot 4(0 \cdot 7-4 \cdot 9) \\
\mathbf{1} \cdot \mathbf{1}(\mathbf{1} \cdot \mathbf{0}-\mathbf{4} \cdot \mathbf{1})\end{array}$ \\
\hline & All adult cestodes & $\begin{array}{l}1999 \\
2002 \\
\text { combined }\end{array}$ & $\begin{array}{l}22 \cdot 5(12 \cdot 3-38 \cdot 6) \\
48 \cdot 6(37 \cdot 4-60 \cdot 6) \\
\mathbf{3 9 \cdot 3}(\mathbf{3 1} \cdot \mathbf{1}-\mathbf{4 9 \cdot 4})\end{array}$ & $\begin{array}{l}4 \cdot 9(1 \cdot 2-17 \cdot 2) \\
28 \cdot 8(19 \cdot 7-40 \cdot 4) \\
20 \cdot 2(14 \cdot 3-29 \cdot 3)\end{array}$ & $\begin{array}{l}1 \cdot 7(0 \cdot 2-9 \cdot 7) \\
2 \cdot 7(0 \cdot 7-9 \cdot 7) \\
\mathbf{2} \cdot \mathbf{3}(\mathbf{1} \cdot \mathbf{1}-\mathbf{7} \cdot \mathbf{4})\end{array}$ & $\begin{array}{l}8 \cdot 6(5 \cdot 2-15 \cdot 0) \\
26 \cdot 5(21 \cdot 5-33 \cdot 3) \\
\mathbf{1 9} \cdot \mathbf{6}(\mathbf{1 6} \cdot \mathbf{2 - 2 4} \cdot \mathbf{4})\end{array}$ \\
\hline & $\begin{array}{l}\text { Mesocestoides } \\
\text { lineatus }\end{array}$ & $\begin{array}{l}1999 \\
2002 \\
\text { combined }\end{array}$ & $\begin{array}{l}2 \cdot 5(0 \cdot 3-14 \cdot 3) \\
4 \cdot 2(1 \cdot 7-12 \cdot 8) \\
\mathbf{3} \cdot \mathbf{6}(\mathbf{1} \cdot \mathbf{5}-\mathbf{9} \cdot \mathbf{4})\end{array}$ & $\begin{array}{l}2 \cdot 4(0 \cdot 3-14 \cdot 0) \\
1 \cdot 4(0 \cdot 3-8 \cdot 5) \\
\mathbf{1} \cdot \mathbf{8}(\mathbf{0} \cdot \mathbf{4}-\mathbf{6} \cdot \mathbf{7})\end{array}$ & $\begin{array}{l}1 \cdot 7(0 \cdot 2-9 \cdot 7) \\
1 \cdot 4(0 \cdot 3-8 \cdot 5) \\
\mathbf{1} \cdot \mathbf{5}(\mathbf{0} \cdot \mathbf{5}-\mathbf{6} \cdot \mathbf{2})\end{array}$ & $\begin{array}{l}2 \cdot 2(1 \cdot 1-7 \cdot 3) \\
2 \cdot 3(1 \cdot 4-6 \cdot 2) \\
2 \cdot 2(\mathbf{1} \cdot \mathbf{7}-\mathbf{5} \cdot \mathbf{3})\end{array}$ \\
\hline & Taenia martis & $\begin{array}{l}1999 \\
2002 \\
\text { combined }\end{array}$ & $\begin{array}{l}0(0-12 \cdot 3) \\
12 \cdot 5(6 \cdot 8-22 \cdot 6) \\
\mathbf{8} \cdot 0(\mathbf{4} \cdot \mathbf{8}-\mathbf{1 5} \cdot 8)\end{array}$ & $\begin{array}{l}0(0-11 \cdot 9) \\
0(0-6 \cdot 7) \\
\mathbf{0}(\mathbf{0 - 4 \cdot 9 )}\end{array}$ & $\begin{array}{l}1 \cdot 7(0 \cdot 2-9 \cdot 7) \\
1 \cdot 4(0 \cdot 3-8 \cdot 5) \\
\mathbf{0} \cdot \mathbf{8}(\mathbf{0} \cdot \mathbf{1}-\mathbf{4} \cdot \mathbf{5})\end{array}$ & $\begin{array}{l}0 \cdot 7(0 \cdot 1-4 \cdot 4) \\
4 \cdot 6(2 \cdot 8-8 \cdot 7) \\
\mathbf{3} \cdot \mathbf{1}(2 \cdot 4-6 \cdot 5)\end{array}$ \\
\hline & Taenia mustelae & $\begin{array}{l}1999 \\
2002 \\
\text { combined }\end{array}$ & $\begin{array}{l}2 \cdot 5(0 \cdot 3-14 \cdot 3) \\
4 \cdot 2(1 \cdot 7-12 \cdot 8) \\
\mathbf{3} \cdot \mathbf{6}(\mathbf{1} \cdot \mathbf{5}-\mathbf{9} \cdot \mathbf{4})\end{array}$ & $\begin{array}{l}0(0-11 \cdot 9) \\
4 \cdot 1(1 \cdot 7-12 \cdot 8) \\
2 \cdot 6(\mathbf{1} \cdot \mathbf{0}-\mathbf{7} \cdot \mathbf{9})\end{array}$ & $\begin{array}{l}3 \cdot 4(0 \cdot 9-12 \cdot 6) \\
2 \cdot 7(0 \cdot 7-9 \cdot 7) \\
\mathbf{3} \cdot \mathbf{0}(\mathbf{1} \cdot \mathbf{6}-\mathbf{8} \cdot \mathbf{8})\end{array}$ & $\begin{array}{l}2 \cdot 2(1 \cdot 1-7 \cdot 3) \\
3 \cdot 7(2 \cdot 0-7 \cdot 4) \\
\mathbf{3} \cdot \mathbf{1}(\mathbf{2} \cdot \mathbf{4}-\mathbf{6} \cdot \mathbf{5})\end{array}$ \\
\hline & $\begin{array}{l}\text { Cladotaenia } \\
\text { globifera }\end{array}$ & $\begin{array}{l}1999 \\
2002 \\
\text { combined }\end{array}$ & $\begin{array}{l}0(0-12 \cdot 3) \\
1 \cdot 4(0 \cdot 3-8 \cdot 6) \\
\mathbf{0} \cdot \mathbf{9}(\mathbf{0} \cdot \mathbf{1}-\mathbf{5} \cdot \mathbf{0})\end{array}$ & $\begin{array}{l}0(0-11 \cdot 9) \\
0(0-6 \cdot 7) \\
\mathbf{0}(\mathbf{0}-\mathbf{4} \cdot \mathbf{9})\end{array}$ & $\begin{array}{l}0(0-8 \cdot 0) \\
1 \cdot 4(0 \cdot 3-8 \cdot 5) \\
\mathbf{0} \cdot \mathbf{8}(\mathbf{0} \cdot \mathbf{1}-\mathbf{4} \cdot \mathbf{5})\end{array}$ & $\begin{array}{l}0(0-4 \cdot 4) \\
0 \cdot 9(0 \cdot 2-3 \cdot 5) \\
\mathbf{0} \mathbf{6}(\mathbf{( 0 \cdot 3 - 2 \cdot 7 )}\end{array}$ \\
\hline & $\begin{array}{r}\text { All larval } \\
\text { cestodes }\end{array}$ & $\begin{array}{l}1999 \\
2002 \\
\text { combined }\end{array}$ & $\begin{array}{l}\mathbf{5} \cdot 0(1 \cdot 2-17 \cdot 5) \\
20 \cdot 8(13 \cdot 0-31 \cdot 9) \\
\mathbf{1 5} \cdot \mathbf{2}(\mathbf{1 0} \cdot \mathbf{2}-\mathbf{2 3} \cdot \mathbf{8})\end{array}$ & $\begin{array}{l}2 \cdot 4(0 \cdot 3-14 \cdot 0) \\
5 \cdot 5(2 \cdot 3-14 \cdot 0) \\
\mathbf{4} \cdot \mathbf{4}(\mathbf{2} \cdot \mathbf{2}-\mathbf{1 0} \cdot \mathbf{7})\end{array}$ & $\begin{array}{l}6 \cdot 9(2 \cdot 5-16 \cdot 7) \\
5 \cdot 4(2 \cdot 3-13 \cdot 9) \\
\mathbf{6} \cdot \mathbf{1}(\mathbf{3} \cdot \mathbf{7}-\mathbf{1 2} \cdot \mathbf{7})\end{array}$ & $\begin{array}{l}2 \cdot 0(3 \cdot 1-11 \cdot 1) \\
10 \cdot 5(7 \cdot 4-15 \cdot 9) \\
\mathbf{8} \cdot \mathbf{4}(\mathbf{6} \cdot \mathbf{5}-\mathbf{1 2} \cdot \mathbf{4})\end{array}$ \\
\hline & All cestodes & $\begin{array}{l}1999 \\
2002 \\
\text { combined }\end{array}$ & $\begin{array}{l}25 \cdot 0(14 \cdot 0-41 \cdot 2) \\
52 \cdot 8(41 \cdot 4-64 \cdot 2) \\
\mathbf{4 2} \cdot \mathbf{9}(\mathbf{3 4} \cdot \mathbf{0}-\mathbf{5 2} \cdot \mathbf{4})\end{array}$ & $\begin{array}{l}7 \cdot 3(2 \cdot 8-21 \cdot 3) \\
31 \cdot 5(22 \cdot 2-43 \cdot 4) \\
\mathbf{2 2} \cdot 8(\mathbf{1 5} \cdot \mathbf{9}-\mathbf{3 1} \cdot \mathbf{7})\end{array}$ & $\begin{array}{l}8 \cdot 6(3 \cdot 7-19 \cdot 2) \\
8 \cdot 1(4 \cdot 2-17 \cdot 7) \\
\mathbf{8} \cdot \mathbf{3}(\mathbf{5} \cdot \mathbf{1}-\mathbf{1 5} \cdot \mathbf{2})\end{array}$ & $\begin{array}{l}12 \cdot 9(8 \cdot 2-19 \cdot 5) \\
30 \cdot 6(25 \cdot 3-37 \cdot 4) \\
\mathbf{2 3} \cdot \mathbf{7}(\mathbf{1 9} \cdot \mathbf{9}-\mathbf{2 8} \cdot \mathbf{6})\end{array}$ \\
\hline & $\begin{array}{l}\text { All helminths } \\
\text { (nematodes \& } \\
\text { cestodes } \\
\text { combined) }\end{array}$ & $\begin{array}{l}1999 \\
2002 \\
\text { combined }\end{array}$ & $\begin{array}{l}95 \cdot 0(83 \cdot 9-99 \cdot 2) \\
86 \cdot 1(77 \cdot 4-93 \cdot 2) \\
\mathbf{8 9} \cdot \mathbf{3}(\mathbf{8 3} \cdot \mathbf{1}-\mathbf{9 4} \cdot \mathbf{4})\end{array}$ & $\begin{array}{l}68 \cdot 3(53 \cdot 9-81 \cdot 6) \\
76 \cdot 7(65 \cdot 9-85 \cdot 5) \\
\mathbf{7 3} \cdot \mathbf{7}(\mathbf{6 5} \cdot \mathbf{3}-\mathbf{8 1} \cdot \mathbf{4})\end{array}$ & $\begin{array}{l}91 \cdot 4(82 \cdot 2-96 \cdot 9) \\
73 \cdot 0(62 \cdot 0-82 \cdot 1) \\
\mathbf{8 1} \cdot \mathbf{1}(\mathbf{7 4} \cdot \mathbf{5}-\mathbf{8 7} \cdot \mathbf{8})\end{array}$ & $\begin{array}{l}85 \cdot 6(79 \cdot 3-90 \cdot 9) \\
78 \cdot 5(73 \cdot 1-84 \cdot 0) \\
\mathbf{8 1} \cdot \mathbf{3}(\mathbf{7 7} \cdot \mathbf{7 - 8 5} \cdot \mathbf{6})\end{array}$ \\
\hline
\end{tabular}

* Prevalence-\% infected (lower and upper 95\% confidence limits).

Note 1 vole in 2002 had nematode larvae in its liver that could not be identified, but were considered to be a different species to those listed above. 

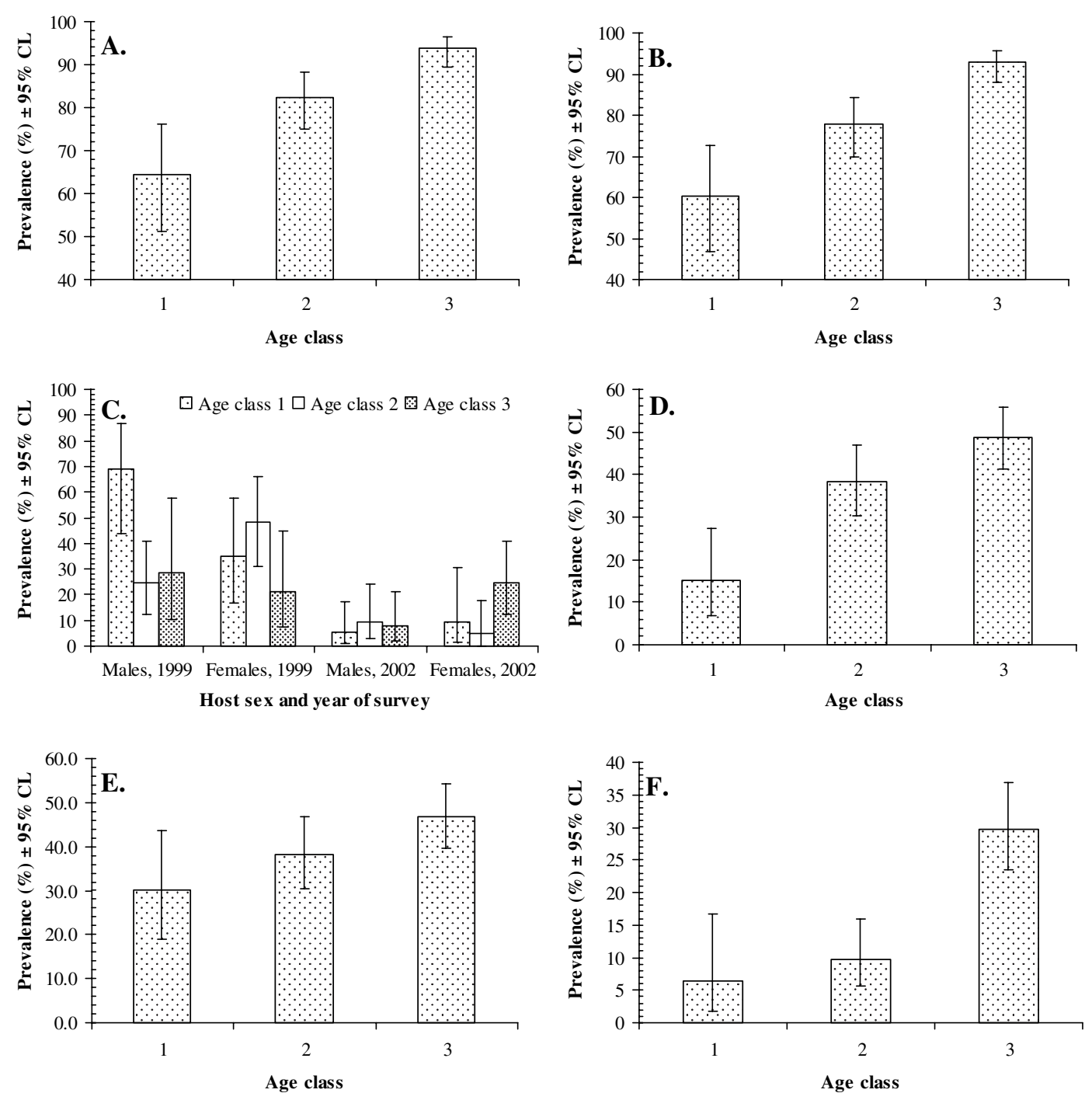

Fig. 3. Age-related effects on the prevalence of helminth parasites. (A) Increasing prevalence of all helminths with increasing host age. (B) Increasing prevalence of all nematodes with increasing host age. (C) Variation in prevalence of $H$. glareoli in the three age classes of voles and in relation to host sex and both surveys. (D) Increasing prevalence of H. mixtum with increasing host age. (E) Increasing prevalence of A. tetraptera with increasing host age. (F) Increasing prevalence of $M$. muris with increasing host age.

At Urwitałt the prevalence of both adult and larval cestodes increased between the two surveys, whereas at Talty the increased prevalence in 2002 was mainly accounted for by adults (Table 3). At Pilchy, the prevalence of both larvae and adults was much the same in both years.

At the species level, the greatest change in prevalence was seen with $C$. henttoneni, which increased by $17 \cdot 2 \%$ (Table 3 ; year $*$ presence/absence of $C$. henttoneni, $\left.\chi^{2}{ }_{1}=18 \cdot 4, P<0 \cdot 0001\right)$. This was evident at all three sites, although most marked at Urwitalt $(25 \cdot 8 \%)$ and Talty $(22 \cdot 5 \%)$. At Pilchy this species remained relatively rare. The difference in prevalence between sites was significant but was confounded by host sex and age, as illustrated in Fig. 3C (site*sex*age* presence/absence of $C$. henttoneni, $\chi^{2}{ }_{4}=18 \cdot 03, P=0 \cdot 0012$ ). Prevalence was numerically higher in males, but there was no independent significant effect of host sex (males $=21 \cdot 5 \%$ [14.7$30 \cdot 5]$ and females $=15 \cdot 1 \%[9 \cdot 4-22 \cdot 8])$.

The remaining cestodes were not analysed further because of their low prevalence, but a few subjective observations are justified. P. omphalodes was present at Urwitalt in both surveys with a similar prevalence and absent on both occasions at Pilchy. It was found in a single vole at Tałty in 2002 but not in 1999 . $M$. lineatus was present in all three sites and showed a very similar low prevalence in both years. T. martis was absent from Talty, showed a similar prevalence at Pilchy in both surveys, and appeared at Urwitałt in 2002, where it had not been detected in 1999. In contrast, T. mustelae showed similar prevalence at both Urwitalt and Pilchy in both surveys, and appeared at Tałty in 2002. C. globifera was not detected at all in 1999, but was present in 1 vole each at Urwitalt and Pilchy in 2002. 

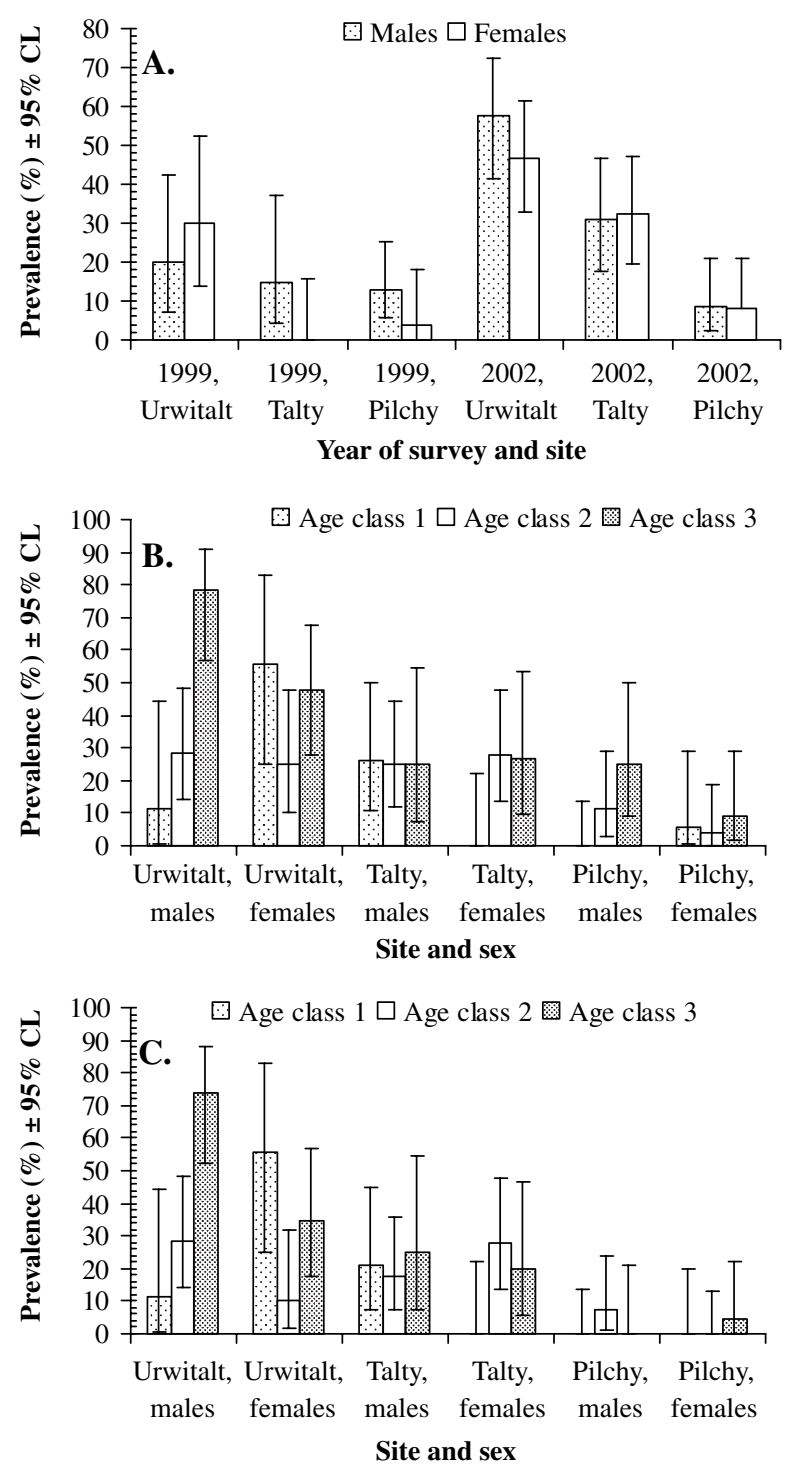

Fig. 4. Combinations of factors affecting the prevalence of cestodes. (A) Variation in prevalence of all cestodes between male and female voles, from three sites and in both surveys. (B) Variation in prevalence of all cestodes among three age classes, in voles of both sexes and from the three study sites. (C) Variation in prevalence of $C$. henttoneni among three age classes, in voles of both sexes and from the three study sites.

\section{Frequency distributions and measures of aggregation}

Of the 14 species of helminths recovered, only 5 species (4 nematodes and 1 cestode) had an overall prevalence greater than $10 \%$ (Table 3 ) and quantitative analyses were confined to these species, with $S$. petrusewiczi. The latter was included because in some subsets of data it showed high prevalence, even though its overall prevalence was only $7 \%$, and because the Berger-Parker dominance index identified this species as dominant more often than others (accompanying paper, Behnke et al. 2008).

Frequency distributions were fitted to all species for which quantitative data were available, by site, by year and in relevant combinations, and were tested for goodness of fit to the positive and negative binomial and Poisson distributions. As can be seen from Table 4, the parameters calculated mostly indicated good approximations to the negative binomial. In most cases $D$ was well above $0 \cdot 6$. The exceptions were H. mixtum in 1999 at Urwitalt, the combined data-set for this site (but in the latter case a test for goodness of fit to the negative binomial failed to reject this distribution) and H. glareoli in 1999 at Pilchy. With the single exception of $H$. glareoli at Urwitalt in 1999 and in the combined data-set for that site, the index of dispersion was greater than or equal to 1 , and mostly considerably higher, ranging to 3945 for S. petrusewiczi in 1999 at Urwitałt. For the frequency distribution of $M$. muris at Urwitalt in 1999, C. henttoneni at all sites combined in 1999 and Tałty and Pilchy in both years, Urwitałt in 1999 and Talty in 2002, we could not reject positive or Poisson distributions, but in all other cases they were either rejected or could not be fitted because of insufficient degrees of freedom. Normal distributions could not be rejected for $H$. mixtum at Talty, for $H$. glareoli at Pilchy and for C. henttoneni at Urwitalt in 1999, for $C$. henttoneni at Talty in 2002 and in both years combined and for A. tetraptera in both 1999 and 2002 at Pilchy, in Pilchy both years combined and at Talty in 2002 .

\section{Abundance of infection}

Analysis of the abundance of $H$. mixtum was conducted on a model excluding sex, because when we included sex the models would not converge. A model including all the main effects without interactions, indicated that sex was not significant (model with negative binomial errors, main effect of sex, $\left.L R_{1}=0 \cdot 04, P=0 \cdot 85\right)$.

Table 3 shows that $H$. mixtum was totally absent from Pilchy in both years and Table 5 shows that abundance was higher at Urwitalt compared to Tałty in both years (main effect of site, $L R_{2}=187 \cdot 7$, $P<0 \cdot 0001)$. Abundance fell significantly between 1999 and 2002 (main effect of year, $L R_{1}=21 \cdot 6$, $P<0 \cdot 0001)$, but proportionally the reduction was greater at Urwitalt, where infections were heavier in 1999 (Table 5; 2-way interaction site $*$ year, $L R_{2}=$ $10 \cdot 7, P=0 \cdot 005)$.

There was a significant main effect of age $\left(L R_{2}=19 \cdot 8, P<0 \cdot 0001\right)$, abundance of $H$. mixtum increasing with age (mean worm burdens in age class $1=0 \cdot 9 \pm 0 \cdot 34$, age class $2=2 \cdot 2 \pm 0 \cdot 28$ and age class $3=2 \cdot 9 \pm 0 \cdot 38$ ), but this was confounded by a significant 2-way interaction with year of study $\left(L R_{2}=17 \cdot 3, P=0 \cdot 0002\right)$. Since no voles at Pilchy carried this species, the interaction between year and age is illustrated only for voles from the other two sites (Fig. 5A). This shows that abundance was consistently higher in 1999 and that in relative terms, despite lower overall abundance in 2002, the rate of 
Table 4. Measures of aggregation for individual species of nematodes by site

\begin{tabular}{|c|c|c|c|c|c|c|c|c|c|c|c|c|c|}
\hline \multirow[b]{2}{*}{ Species } & \multirow[b]{2}{*}{ Year } & \multicolumn{3}{|c|}{ Urwitałt } & \multicolumn{3}{|l|}{ Tałty } & \multicolumn{3}{|l|}{ Pilchy } & \multicolumn{3}{|c|}{ Combined } \\
\hline & & $k^{1}$ & $I^{2}$ & $D^{3}$ & $k^{1}$ & $I^{2}$ & $D^{3}$ & $k^{1}$ & $I^{2}$ & $D^{3}$ & $k^{1}$ & $I^{2}$ & $D^{3}$ \\
\hline $\begin{array}{l}\text { Heligmosomum } \\
\text { mixtum }\end{array}$ & $\begin{array}{l}1999 \\
2002 \\
\text { Combined }\end{array}$ & $\begin{array}{l}3 \cdot 53 \\
0 \cdot 90^{*} \\
\mathbf{0} \cdot 98^{*}\end{array}$ & $\begin{array}{l}2 \cdot 43 \\
2 \cdot 96 \\
\mathbf{3} \cdot \mathbf{6 8}\end{array}$ & $\begin{array}{l}0 \cdot 36 \\
0 \cdot 62 \\
\mathbf{0} \cdot 56\end{array}$ & $\begin{array}{l}0 \cdot 52^{*} \\
0 \cdot 26^{*} \\
\mathbf{0} \cdot \mathbf{3 3} \text { * }\end{array}$ & $\begin{array}{l}2 \cdot 56 \\
4 \cdot 16 \\
\mathbf{3} \cdot \mathbf{5 0}\end{array}$ & $\begin{array}{l}0 \cdot 67 \\
0 \cdot 79 \\
\mathbf{0} \cdot 76\end{array}$ & $\begin{array}{l}\mathrm{nd}^{4} \\
\mathrm{nd} \\
\text { nd }\end{array}$ & $\begin{array}{l}\text { nd } \\
\text { nd } \\
\text { nd }\end{array}$ & $\begin{array}{l}\text { nd } \\
\text { nd } \\
\text { nd }\end{array}$ & $\begin{array}{l}0 \cdot 26 \text { ฯ } \\
0 \cdot 26 \text { * } \\
\mathbf{0} \cdot \mathbf{2 4}\end{array}$ & $\begin{array}{l}5 \cdot 25 \\
3 \cdot 95 \\
\mathbf{4} \cdot 87\end{array}$ & $\begin{array}{l}0 \cdot 74 \\
0 \cdot 81 \\
\mathbf{0} \cdot \mathbf{7 9}\end{array}$ \\
\hline $\begin{array}{l}\text { Heligmosomoides } \\
\text { glareoli }\end{array}$ & $\begin{array}{l}1999 \\
2002 \\
\text { Combined }\end{array}$ & $\begin{array}{l}\text { nd } \\
\text { nd } \\
\text { nd }\end{array}$ & $\begin{array}{l}0 \cdot 95 \\
1 \cdot 00 \\
\mathbf{0 \cdot 9 7}\end{array}$ & $\begin{array}{l}0 \cdot 90 \\
0 \cdot 97 \\
\mathbf{0 \cdot 9 6}\end{array}$ & $\begin{array}{l}\text { nd } \\
\text { nd } \\
\text { nd }\end{array}$ & $\begin{array}{l}1 \cdot 00 \\
1 \cdot 00 \\
\mathbf{0} \cdot 99\end{array}$ & $\begin{array}{l}0 \cdot 95 \\
0 \cdot 97 \\
\mathbf{0} \cdot 97\end{array}$ & $\begin{array}{l}1 \cdot 20 * \\
0 \cdot 20 \text { ฯ } \\
\mathbf{0} \cdot 399\end{array}$ & $\begin{array}{l}3 \cdot 62 \\
4 \cdot 10 \\
4 \cdot 85\end{array}$ & $\begin{array}{l}0 \cdot 48 \\
0 \cdot 80 \\
\mathbf{0} \cdot 68\end{array}$ & $\begin{array}{l}0 \cdot 20 \text { ฯ } \\
0 \cdot 06^{*} \\
\mathbf{0} \cdot \mathbf{1 0}\end{array}$ & $\begin{array}{l}6 \cdot 02 \\
4 \cdot 60 \\
6 \cdot 26\end{array}$ & $\begin{array}{l}0 \cdot 77 \\
0 \cdot 93 \\
\mathbf{0} \cdot \mathbf{8 8}\end{array}$ \\
\hline $\begin{array}{l}\text { Syphacia } \\
\text { petrusewiczi }\end{array}$ & $\begin{array}{l}1999 \\
2002 \\
\text { Combined }\end{array}$ & $\begin{array}{l}0 \cdot 02 \\
0 \cdot 01 \\
\mathbf{0} \cdot 01\end{array}$ & $\begin{array}{l}3945 \\
316 \cdot 1 \\
\mathbf{3 4 2 6}\end{array}$ & $\begin{array}{l}0 \cdot 95 \\
0 \cdot 96 \\
\mathbf{0} \cdot 97\end{array}$ & $\begin{array}{l}0 \cdot 05^{*} \\
\text { nd } \\
\mathbf{0} \cdot 02 *\end{array}$ & $\begin{array}{l}1847 \\
1 \cdot 00 \\
1892\end{array}$ & $\begin{array}{l}0 \cdot 90 \\
0 \cdot 97 \\
\mathbf{0} \cdot 96\end{array}$ & $\begin{array}{l}0 \cdot 03 \\
\text { nd } \\
\mathbf{0} \cdot 01\end{array}$ & $\begin{array}{l}2 \cdot 47 \\
\text { nd } \\
2 \cdot 50\end{array}$ & $\begin{array}{l}0 \cdot 96 \\
\text { nd } \\
\mathbf{0 \cdot 9 8}\end{array}$ & $\begin{array}{l}0 \cdot 02 * \\
0 \cdot 01 \\
\mathbf{0} \cdot 01\end{array}$ & $\begin{array}{l}2816 \\
318 \cdot 9 \\
2660\end{array}$ & $\begin{array}{l}0.97 \\
0.99 \\
\mathbf{0 . 9 8}\end{array}$ \\
\hline $\begin{array}{r}\text { Aspiculuris } \\
\text { tetraptera }\end{array}$ & $\begin{array}{l}1999 \\
2002 \\
\text { Combined }\end{array}$ & $\begin{array}{l}\text { nd } \\
0 \cdot 08^{*} \\
\mathbf{0} \cdot 05^{*}\end{array}$ & $\begin{array}{l}1 \\
63 \cdot 14 \\
\mathbf{6 3} \cdot \mathbf{0 8}\end{array}$ & $\begin{array}{l}0 \cdot 95 \\
0 \cdot 93 \\
\mathbf{0 . 9 5}\end{array}$ & $\begin{array}{l}0 \cdot 02 \\
0 \cdot 30^{*} \\
\mathbf{0} \cdot \mathbf{1 3} \text { * }\end{array}$ & $\begin{array}{l}184 \cdot 3 \\
14 \cdot 24 \\
\mathbf{1 1 2} \cdot 3\end{array}$ & $\begin{array}{l}0 \cdot 93 \\
0 \cdot 76 \\
\mathbf{0} \cdot 89\end{array}$ & $\begin{array}{l}0 \cdot 21 * \\
0 \cdot 30 \text { ฯ } \\
\mathbf{0} \cdot \mathbf{2 4}\end{array}$ & $\begin{array}{l}81 \cdot 84 \\
56 \cdot 24 \\
74 \cdot 36\end{array}$ & $\begin{array}{l}0 \cdot 81 \\
0 \cdot 78 \\
\mathbf{0} \cdot 82\end{array}$ & $\begin{array}{l}0 \cdot 07 \text { * } \\
0 \cdot 21 * \\
\mathbf{0} \cdot \mathbf{1 3}\end{array}$ & $\begin{array}{l}117 \cdot 7 \\
43 \cdot 27 \\
\mathbf{8 7} \cdot \mathbf{0 3}\end{array}$ & $\begin{array}{l}0 \cdot 92 \\
0 \cdot 84 \\
\mathbf{0} \cdot 89\end{array}$ \\
\hline $\begin{array}{l}\text { Mastophorus } \\
\text { muris }\end{array}$ & $\begin{array}{l}1999 \\
2002 \\
\text { Combined }\end{array}$ & $\begin{array}{l}0 \cdot 17 \\
0 \cdot 19^{*} \\
\mathbf{0} \cdot 14^{*}\end{array}$ & $\begin{array}{l}1 \cdot 56 \\
8 \cdot 75 \\
8 \cdot 68\end{array}$ & $\begin{array}{l}0 \cdot 89 \\
0 \cdot 83 \\
\mathbf{0} \cdot 87\end{array}$ & $\begin{array}{l}\text { nd } \\
\text { nd } \\
\text { nd }\end{array}$ & $\begin{array}{l}\text { nd } \\
1 \cdot 00 \\
\mathbf{1} \cdot 00\end{array}$ & $\begin{array}{l}\text { nd } \\
0 \cdot 97 \\
\mathbf{0 . 9 8}\end{array}$ & $\begin{array}{l}0 \cdot 13^{*} \\
0 \cdot 07^{*} \\
\mathbf{0} \cdot 08^{*}\end{array}$ & $\begin{array}{l}4 \cdot 65 \\
18 \cdot 02 \\
\mathbf{1 5} \cdot \mathbf{7 6}\end{array}$ & $\begin{array}{l}0 \cdot 89 \\
0 \cdot 91 \\
\mathbf{0} \cdot 92\end{array}$ & $\begin{array}{l}0 \cdot 08^{*} \\
0 \cdot 08^{*} \\
\mathbf{0} \cdot 07^{*}\end{array}$ & $\begin{array}{l}4 \cdot 16 \\
13 \cdot 94 \\
\mathbf{1 2} \cdot 87\end{array}$ & $\begin{array}{l}0 \cdot 94 \\
0 \cdot 92 \\
\mathbf{0} \cdot 94\end{array}$ \\
\hline $\begin{array}{l}\text { Catenotaenia } \\
\text { henttoneni }\end{array}$ & $\begin{array}{l}1999 \\
2002 \\
\text { Combined }\end{array}$ & $\begin{array}{l}0 \cdot 55^{*} \\
0 \cdot 37^{*} \\
\mathbf{0} \cdot 29^{*}\end{array}$ & $\begin{array}{l}1 \cdot 49 \\
5 \cdot 66 \\
\mathbf{5 \cdot 5 6}\end{array}$ & $\begin{array}{l}0 \cdot 82 \\
0 \cdot 75 \\
\mathbf{0} \cdot 80\end{array}$ & $\begin{array}{l}\text { nd } \\
0 \cdot 61 * \\
\mathbf{0} \cdot 39 *\end{array}$ & $\begin{array}{l}0 \cdot 98 \\
1 \cdot 95 \\
\mathbf{1} \cdot 99\end{array}$ & $\begin{array}{l}0 \cdot 93 \\
0 \cdot 79 \\
\mathbf{0} \cdot 85\end{array}$ & $\begin{array}{l}\text { nd } \\
0 \cdot 02 \\
\mathbf{0 \cdot 0 2}\end{array}$ & $\begin{array}{l}1 \cdot 00 \\
2 \cdot 57 \\
2 \cdot 31\end{array}$ & $\begin{array}{l}0 \cdot 97 \\
0 \cdot 96 \\
\mathbf{0 \cdot 9 7}\end{array}$ & $\begin{array}{l}0 \cdot 21^{*} \\
0 \cdot 20^{*} \\
\mathbf{0} \cdot \mathbf{1 5} \text { * }\end{array}$ & $\begin{array}{l}1 \cdot 48 \\
5 \cdot 27 \\
\mathbf{5} \cdot \mathbf{0 8}\end{array}$ & $\begin{array}{l}0 \cdot 93 \\
0 \cdot 86 \\
\mathbf{0} \cdot 89\end{array}$ \\
\hline
\end{tabular}

1. Negative binomial exponent.

2. Index of dispersion $=$ Variance to mean ratio.

3. Index of discrepancy (Poulin, 1993).

4. Not possible to calculate parameters (too few infected animals).

* Not significantly different from the negative binomial.

- Significantly different from the negative binomial.

Note that where no symbol is shown, it was not possible to test for significance because of too few degrees of freedom, or too few voles were infected.

increase from age class 1 to 3 was greater than in 1999.

Statistical analysis of the abundance of $H$. glareoli yielded a simple model with just 2 highly significant terms. There was a significant difference between sites (Table $5, L R_{2}=151 \cdot 2, P<0 \cdot 0001$ ) with this species being predominantly encountered at Pilchy, and only occasionally occurring in voles from the other two sites. This was the case in both years, although in 2002, overall abundance fell quite markedly (main effect of year, $L R_{1}=29 \cdot 5, P<0 \cdot 0001$ ).

M. muris was virtually absent from Tałty in both years, only 1 worm being detected in 2002, so the site effect was highly significant (Table 5 ; main effect of site, $\left.L R_{2}=35 \cdot 2, P<0 \cdot 0001\right)$. Abundance was similar at Urwitalt and Pilchy (Table 5), and whilst in both it increased from 1999 to 2002, when other factors were taken into consideration this between-year change was just outside our cut-off for significance (main effect of year, $L R_{1}=3 \cdot 2, P=0 \cdot 073$ ). To some extent it was confounded by a significant 2 -way interaction (year $*$ site, $L R_{2}=9 \cdot 5, P=0 \cdot 008$ ), arising mainly because at Talty the increase was from zero in 1999 to just 0.01 in 2002, whilst at Urwitalt and Pilchy abundance increased more markedly (Table 5, 9.3and $3 \cdot 5$-fold increases, respectively).

There was a highly significant main effect of age (Fig. $5 \mathrm{~B}, L R_{2}=21 \cdot 5, P<0 \cdot 0001$ ), abundance increasing with age in both years although the relative increase was more prominent in 2002 (but the 2 -way interaction year $*$ age was just outside significance $L R_{2}=5 \cdot 71, P=0 \cdot 058$ ). There was also a significant 2 -way interaction site $*$ age (not illustrated, $\left.L R_{4}=10 \cdot 3, P=0 \cdot 035\right)$. In this species abundance is usually higher in females (main effect of sex, $\left.L R_{1}=9 \cdot 85, P=0 \cdot 0017\right)$, and this was the case in both years (Fig. 5D in 1999 and 2002 mean worm burdens were 3.6 and 4.9 times higher, respectively in females compared with males). Fig. 5C, based only on data from Urwitalt and Pilchy, where the parasite was most prevalent, shows that the abundance of $M$. muris was already marginally higher in female age class 2 voles, but was highest in the oldest animals. There were no higher order interactions in this case.

For S. petrusewiczi, neither full factorial models with negative binomial error structures nor simpler models with only main effects converged satisfactorily in R. Therefore, we used a Box-Cox transformation of the data and fitted a model with a normal error structure (Box-Cox lambda $\pm \mathrm{CL}=$ $-4 \cdot 22450 \pm-4 \cdot 20088 \&-4 \cdot 24813$; Likelihood $=$ $581 \cdot 434)$. Abundance varied significantly between sites and as Table 5 shows $S$. petrusewicz $i$ was rare in both years at Pilchy but more abundant in both at Urwitalt and Talty (main effect of site, $F_{2,353}=7 \cdot 19$, 
Table 5. Abundance of helminth taxa by site and year

\begin{tabular}{|c|c|c|c|c|c|c|}
\hline \multirow[b]{2}{*}{ Taxon } & \multirow[b]{2}{*}{ Species } & \multirow[b]{2}{*}{ Year } & \multicolumn{3}{|l|}{ Site } & \multirow[b]{2}{*}{ Total } \\
\hline & & & Urwitałt* & Tałty* & Pilchy* & \\
\hline \multirow[t]{24}{*}{ Nematodes } & Heligmosomum & 1999 & $5 \cdot 60(0 \cdot 58)$ & $1 \cdot 24(0 \cdot 28)$ & 0 & $1 \cdot 98(0 \cdot 27)$ \\
\hline & mixtum & 2002 & $1 \cdot 76(0 \cdot 27)$ & $1 \cdot 05(0 \cdot 25)$ & 0 & $0.93(0.13)$ \\
\hline & & combined & $3 \cdot 13(0 \cdot 32)$ & $1 \cdot 12(0 \cdot 19)$ & $\mathbf{0}$ & $1 \cdot 34(0 \cdot 13)$ \\
\hline & Heligmosomoides & 1999 & $0 \cdot 08(0 \cdot 04)$ & $0.02(0.02)$ & $4 \cdot 38(0 \cdot 52)$ & $1 \cdot 86(0 \cdot 28)$ \\
\hline & glareoli & 2002 & $0 \cdot 01(0 \cdot 01)$ & $0 \cdot 01(0 \cdot 01)$ & $0.99(0 \cdot 23)$ & $0 \cdot 34(0.09)$ \\
\hline & & combined & $0.04(0.02)$ & $0.02(0.01)$ & $2 \cdot 48(0 \cdot 30)$ & $0.93(0 \cdot 13)$ \\
\hline & Syphacia & 1999 & $102 \cdot 7(100 \cdot 6)$ & $115 \cdot 41(72 \cdot 10)$ & $0 \cdot 07(0 \cdot 05)$ & $63 \cdot 6(35 \cdot 9)$ \\
\hline & petrusewiczi & 2002 & $9 \cdot 35(6 \cdot 41)$ & $0 \cdot 01(0 \cdot 01)$ & & $3 \cdot 08(2 \cdot 12)$ \\
\hline & & combined & $42 \cdot 67(36 \cdot 13)$ & $41 \cdot 52(26 \cdot 25)$ & $0 \cdot 03(0 \cdot 2)$ & $26 \cdot 58(14 \cdot 06)$ \\
\hline & Aspiculuris & 1999 & $0 \cdot 03(0 \cdot 02)$ & $7 \cdot 90(5 \cdot 96)$ & $10 \cdot 98(3 \cdot 94)$ & $6 \cdot 92(2 \cdot 42)$ \\
\hline & tetraptera & 2002 & $1.99(1.32)$ & $3 \cdot 21(0 \cdot 79)$ & $4 \cdot 22(1 \cdot 79)$ & $3 \cdot 15(0 \cdot 79)$ \\
\hline & & combined & $1 \cdot 29(0 \cdot 85)$ & $4 \cdot 89(2 \cdot 20)$ & $7 \cdot 19(2 \cdot 01)$ & $4 \cdot 61(1 \cdot 06)$ \\
\hline & Mastophorus & 1999 & $0 \cdot 15(0 \cdot 08)$ & 0 & $0 \cdot 43(0 \cdot 19)$ & $0 \cdot 22(0 \cdot 08)$ \\
\hline & muris & 2002 & $1 \cdot 39(0 \cdot 41)$ & $0 \cdot 01(0 \cdot 01)$ & $1 \cdot 51(0 \cdot 61)$ & $0.97(0.25)$ \\
\hline & & combined & $0.95(0.27)$ & $0.01(0.01)$ & $1 \cdot 04(0 \cdot 35)$ & $0 \cdot 68(0 \cdot 16)$ \\
\hline & Aonchotheca & 1999 & 0 & 0 & 0 & 0 \\
\hline & annulosa & 2002 & $0 \cdot 26(0 \cdot 16)$ & $0 \cdot 08(0 \cdot 06)$ & 0 & $0 \cdot 11(0 \cdot 06)$ \\
\hline & & combined & $0 \cdot 17(1 \cdot 0)$ & $0.05(0.04)$ & $\mathbf{0}$ & $0.07(0.03)$ \\
\hline & Trichuris spp. & 1999 & 0 & 0 & $0 \cdot 02(0 \cdot 02)$ & $0 \cdot 01(0 \cdot 01)$ \\
\hline & & 2002 & 0 & 0 & & \\
\hline & & combined & 0 & $\mathbf{0}$ & $0.01(0 \cdot 01)$ & $<0.01(<0.01)$ \\
\hline & All nematodes & 1999 & $108 \cdot 5(100 \cdot 6)$ & $124 \cdot 6(72 \cdot 02)$ & $15 \cdot 88(3 \cdot 99)$ & $74 \cdot 60(35 \cdot 87)$ \\
\hline & & 2002 & $14.75(6.52)$ & $4 \cdot 40(0 \cdot 84)$ & $6 \cdot 72(1 \cdot 91)$ & $8 \cdot 58(2 \cdot 27)$ \\
\hline & & combined & $48 \cdot 23(36 \cdot 11)$ & $47 \cdot 62(26 \cdot 27)$ & $10 \cdot 74(2 \cdot 08)$ & $34 \cdot 22(14 \cdot 07)$ \\
\hline \multirow[t]{30}{*}{ Cestodes } & Catenotaenia & 1999 & $0 \cdot 28(0 \cdot 10)$ & $0 \cdot 05(0 \cdot 03)$ & $0 \cdot 02(0 \cdot 02)$ & $0 \cdot 10(0 \cdot 03)$ \\
\hline & henttoneni & 2002 & $1 \cdot 47(0 \cdot 34)$ & $0 \cdot 41(0 \cdot 10)$ & $0.07(0.05)$ & $0 \cdot 64(0 \cdot 13)$ \\
\hline & & combined & $1 \cdot 04(0 \cdot 23)$ & $0.28(0.07)$ & $0.05(0.03)$ & $0.43(0.08)$ \\
\hline & Paranoplocephala & 1999 & $0 \cdot 03(0 \cdot 03)$ & 0 & 0 & $0 \cdot 01(0 \cdot 01)$ \\
\hline & omphalodes & 2002 & $0.03(0.02)$ & $0 \cdot 01(0 \cdot 01)$ & 0 & $0 \cdot 01(0 \cdot 01)$ \\
\hline & & combined & $0.03(0.02)$ & $0.01(0.01)$ & 0 & $0.01(0.01)$ \\
\hline & All adult cestodes & 1999 & $0 \cdot 30(0 \cdot 10)$ & $0 \cdot 05(0 \cdot 03)$ & $0 \cdot 02(0 \cdot 02)$ & $0 \cdot 11(0 \cdot 03)$ \\
\hline & & 2002 & $1 \cdot 50(0 \cdot 34)$ & $0 \cdot 42(0 \cdot 10)$ & $0 \cdot 07(0 \cdot 05)$ & $0 \cdot 66(0 \cdot 12)$ \\
\hline & & combined & $1 \cdot 07(0 \cdot 23)$ & $0 \cdot 29(0 \cdot 07)$ & $0.05(0.03)$ & $0.44(0.08)$ \\
\hline & Mesocestoides & 1999 & $1 \cdot 08(1 \cdot 08)$ & $2 \cdot 27(2 \cdot 27)$ & $0 \cdot 07(0 \cdot 07)$ & $1 \cdot 01(0 \cdot 74)$ \\
\hline & lineatus & 2002 & $0.69(0.52)$ & $5 \cdot 38(5 \cdot 38)$ & $0 \cdot 23(0 \cdot 23)$ & $2 \cdot 10(1 \cdot 80)$ \\
\hline & & combined & $0.83(0.50)$ & $4 \cdot 26(3 \cdot 54)$ & $0 \cdot 16(0 \cdot 13)$ & $1 \cdot 68(1 \cdot 14)$ \\
\hline & Taenia martis & 1999 & 0 & 0 & $0 \cdot 02(0 \cdot 02)$ & $0 \cdot 01(0 \cdot 01)$ \\
\hline & & 2002 & $0 \cdot 14(0 \cdot 05)$ & 0 & $0 \cdot 15(0 \cdot 15)$ & $0.05(0.02)$ \\
\hline & & combined & $0.09(0.03)$ & $\mathbf{0}$ & $0.09(0.08)$ & $0.06(0.03)$ \\
\hline & Taenia mustelae & 1999 & $0 \cdot 03(0 \cdot 02)$ & 0 & $0 \cdot 05(0 \cdot 04)$ & $0 \cdot 03(0 \cdot 02)$ \\
\hline & & 2002 & $0.06(0.03)$ & $0 \cdot 14(0 \cdot 11)$ & $0 \cdot 03(0 \cdot 02)$ & $0 \cdot 12(0 \cdot 06)$ \\
\hline & & combined & $0 \cdot 04(0 \cdot 02)$ & $0.09(0.07)$ & $0.04(0.02)$ & $0.06(0.02)$ \\
\hline & Cladotaenia & 1999 & 0 & 0 & 0 & 0 \\
\hline & globifera & 2002 & $2 \cdot 78(2 \cdot 78)$ & 0 & $3 \cdot 38(3 \cdot 38)$ & $2 \cdot 06(1 \cdot 46)$ \\
\hline & & combined & $1.79(1.79)$ & $\mathbf{0}$ & $1 \cdot 89(1 \cdot 89)$ & $1 \cdot 26(0 \cdot 89)$ \\
\hline & All larval & 1999 & $1 \cdot 10(1 \cdot 07)$ & $2 \cdot 27(2 \cdot 27)$ & $0 \cdot 16(0 \cdot 09)$ & $1 \cdot 05(0 \cdot 74)$ \\
\hline & cestodes & 2002 & $3 \cdot 67(2 \cdot 81)$ & $5 \cdot 52(5 \cdot 38)$ & $3 \cdot 78(3 \cdot 39)$ & $4 \cdot 32(2 \cdot 31)$ \\
\hline & & combined & $2 \cdot 75(1 \cdot 85)$ & $4 \cdot 35(3 \cdot 54)$ & $2 \cdot 19(1 \cdot 90)$ & $3.05(1.44)$ \\
\hline & All cestodes & 1999 & $1 \cdot 40(1 \cdot 07)$ & $2 \cdot 32(2 \cdot 27)$ & $0 \cdot 17(0 \cdot 10)$ & $1 \cdot 16(0 \cdot 74)$ \\
\hline & & 2002 & $5 \cdot 15(2 \cdot 88)$ & $5 \cdot 95(5 \cdot 39)$ & $3 \cdot 85(3 \cdot 39)$ & $4.98(2 \cdot 32)$ \\
\hline & & combined & $3 \cdot 81(1 \cdot 89)$ & $4 \cdot 64(3 \cdot 54)$ & $2 \cdot 23(1 \cdot 90)$ & $3 \cdot 49(1 \cdot 45)$ \\
\hline & All helminths & 1999 & $109 \cdot 9(100 \cdot 5)$ & $126 \cdot 9(72 \cdot 0)$ & $16 \cdot 05(4 \cdot 03)$ & $75 \cdot 75(35 \cdot 86)$ \\
\hline & (nematodes \& & 2002 & $19 \cdot 92(7 \cdot 14)$ & $10 \cdot 34(5 \cdot 49)$ & $10 \cdot 57(3 \cdot 87)$ & $13 \cdot 57(3 \cdot 25)$ \\
\hline & $\begin{array}{l}\text { cestodes } \\
\text { combined) }\end{array}$ & combined & $52 \cdot 05(36 \cdot 13)$ & $52 \cdot 26(26 \cdot 44)$ & $12 \cdot 98(2 \cdot 80)$ & $37 \cdot 71(14 \cdot 13)$ \\
\hline
\end{tabular}

* Mean abundance ( \pm s.E.M. $)$. 


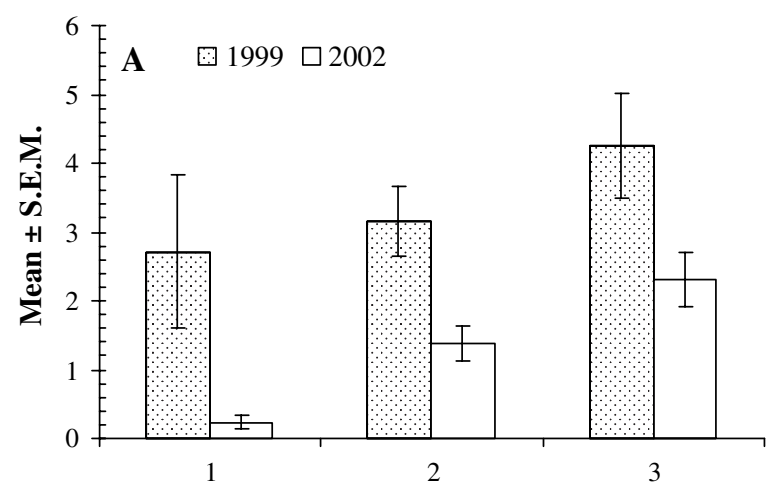

Age Class

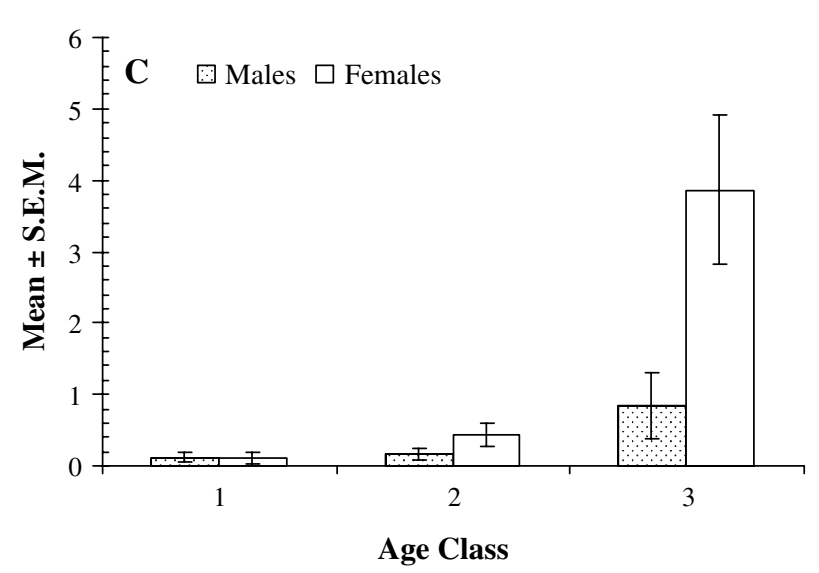

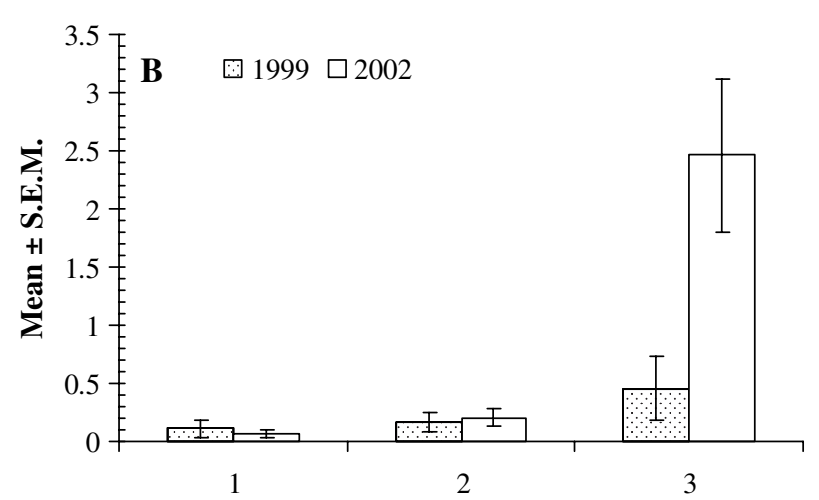

Age Class

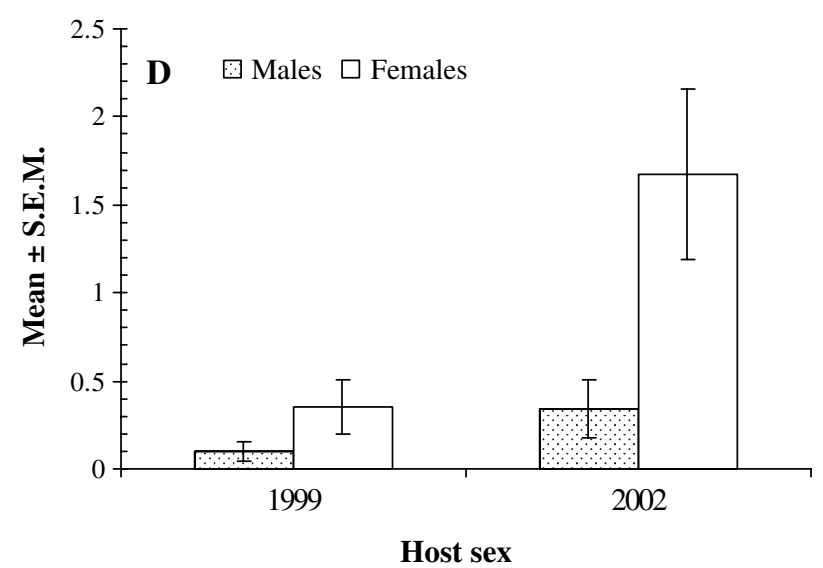

Fig. 5. Factors affecting variation in abundance of H. mixtum and M. muris. (A) Age-dependent increase in abundance of H. mixtum in 1999 and 2002, in two of the sites (Urwitalt and Tałty). Data from Pilchy were excluded because no voles were infected with H. mixtum in either year. (B) Age-dependent increase in abundance of M. muris in 1999 and 2002. Data from all three sites and both sexes combined. (C) Age-dependent increase in abundance of M. muris in male and female voles. Data from Tałty were excluded but both years combined. (D) The effect of host sex on abundance of M. muris in both years. Data from all sites and age classes combined.

$P<0 \cdot 001)$. However, abundance dropped markedly between 1999 and 2002 in all sites (main effect of year, $\left.F_{1,353}=19 \cdot 0, P<0 \cdot 001\right)$ and the relative ranking of Urwitalt and Talty was reversed (2-way interaction between site $*$ year, $\left.F_{2,351}=11 \cdot 4, P<0.001\right)$. Abundance did not differ significantly between age classes nor between sexes. The main effects of year and site were also confirmed by non-parametric tests (Mann-Whitney $\mathrm{U}$ test on year, $z=-3 \cdot 9, P<0 \cdot 001$ and Kruskal Wallis test on site, $\chi^{2}{ }_{2}=11 \cdot 3, P=0 \cdot 004$ ) but no age nor sex effects were evident.

Attempts to fit full factorial models with negative binomial errors to the abundance of $A$. tetraptera (dependent variable) did not converge in R. More restricted models with age, or sex and interactions with age and/or sex, all failed to converge. Therefore we fitted a model with just site and year as main effects and their interaction. As Table 5 shows, in both years abundance of $A$. tetraptera was highest at Pilchy and lowest at Urwitałt (model with negative binomial errors, main effect of site $L R_{2}=14 \cdot 3$, $P=0 \cdot 0008)$. There was also a reduction in abundance between 1999 and 2002 in two sites but a relatively small increase in abundance at Urwitałt (2-way interaction site $*$ year, $\left.L R_{2}=31 \cdot 4, P<0 \cdot 0001\right)$.
Further support for this interpretation came from a 2-way non-parametric analysis of variance, which also supported the strong site effect but also gave a weaker 2 -way interaction and a borderline year effect (main effect of site, $H_{2}=38 \cdot 1, P<0 \cdot 0001$; main effect of year $H_{1}=4 \cdot 12, P=0 \cdot 04$; site $*$ year, $H_{2}=$ $10 \cdot 2, P=0 \cdot 006)$.

To eliminate the possibility of confounding effects from sex and age, we also fitted a model in $\mathrm{R}$ using Box-Cox transformed data, and with a normal error structure (Box-Cox lambda $\pm \mathrm{CI}=-1 \cdot 01858 \pm$ $-1 \cdot 01052 \&-1 \cdot 02665$; Likelihood $=-128 \cdot 248)$. This confirmed the effects of site on $A$. tetraptera $\left(F_{2,352}=36 \cdot 5, P<0 \cdot 0001\right)$ and the interaction between site and year $\left(F_{2,352}=8 \cdot 12, P<0 \cdot 0001\right)$ but indicated that the main effect of year was also significant $\left(F_{1,352}=9 \cdot 3, P=0 \cdot 0024\right)$. Furthermore, the model based on Box-Cox transformed data indicated that abundance varied significantly with age (main effect of age $\left.F_{2,352}=7 \cdot 13, P<0 \cdot 001\right)$ but no clear consistent pattern was evident (Fig. 6). The mean worm burden was highest in age class 1 in 3 data sets (Talty in 1999, Pilchy in 1999 and 2002), in age class 2 in 1 data set (Talty 2002) and in age class 3 in 1 data set (Urwitalt 2002). The significant main effect of age 

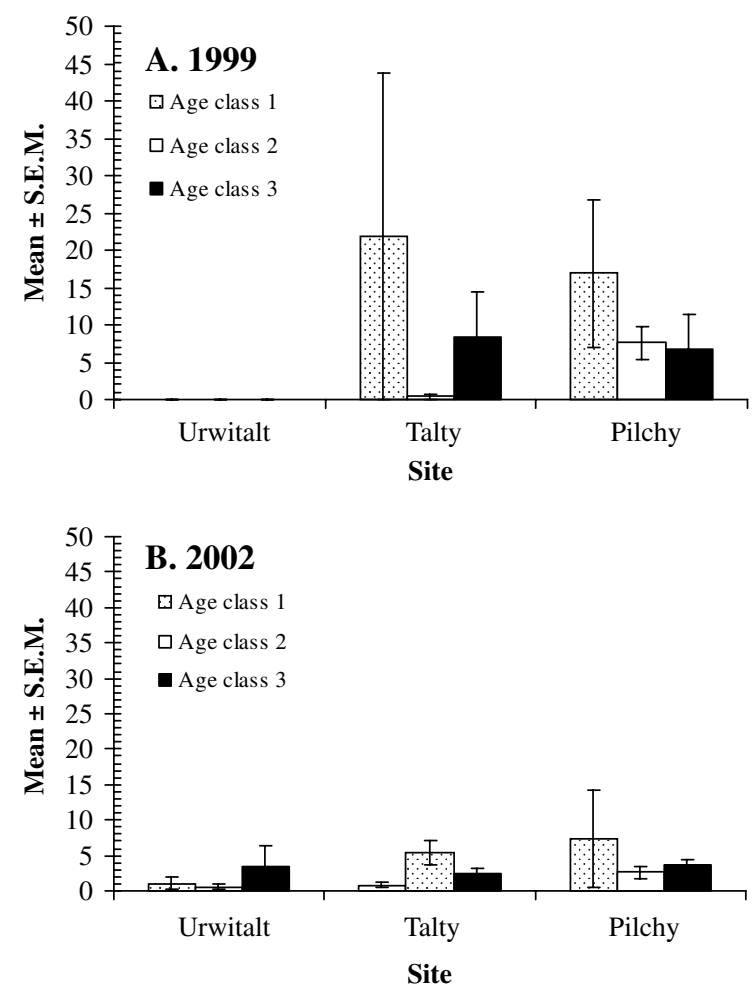

Fig. 6. Variation in the abundance of Aspiculuris tetraptera between sites, year of survey and in age classes. (A) Age-dependent changes in abundance of $A$. tetraptera at the three sites in 1999. (B) Age-dependent changes in abundance of $A$.tetraptera at the three sites in 2002 .

was primarily attributable to the abundance of A. tetraptera being more than twice as high in age class 1 voles $(8 \cdot 5 \pm 3 \cdot 74)$ compared with older animals (age class $2=3 \cdot 0 \pm 0 \cdot 57$; age class $3=3 \cdot 6 \pm 1 \cdot 08$ ).

The strongest effect on $C$. henttoneni was site (model with negative binomial errors, main effect of site $L R_{2}=47 \cdot 8, P<0 \cdot 0001$ ), and as can be seen from Table 5 abundance was highest at Urwitalt and lowest at Pilchy; this was consistent in both years. There was a relatively smaller but significant effect of year (main effect of year $L R_{1}$ stat $=21 \cdot 4, P<0 \cdot 0001$ ). Abundance increased in all sites in 2002, most prominently at Urwitalt and least at Pilchy, although proportionally relative to 1999 the increase was greatest at Tałty (x8.2-fold), compared with Urwitalt (x5.25-fold) and Pilchy (x3.5-fold).

Abundance of $C$. henttoneni also varied significantly with age but not markedly (main effect of age $\left.L R_{2}=6.9, \quad P=0.033\right)$, rising steeply in the oldest voles in the study (age class $1=0 \cdot 30 \pm 0 \cdot 122$, age class $2=0 \cdot 21 \pm 0 \cdot 043$, age class $3=0 \cdot 86 \pm 0 \cdot 219$ ). However, the greater abundance in age class 3 voles was mainly driven by data from Urwitalt, and the same pattern was not obvious at the other two sites, nor in both sexes (2-way interaction age $*$ site, $L R_{4}=9.33, P=0.05 ; 3$-way interaction age $*$ sex $*$ site $\left.L R_{4}=27 \cdot 7, P<0 \cdot 0001\right)$.

None of the remaining parasites were present in sufficient animals to merit analysis.
Table 6. Pooled within-group correlations between discriminating variables and standardized canonical discriminant functions

\begin{tabular}{lrc}
\hline \hline Discriminating variable* & Function 1 & Function 2 \\
\hline Heligmosomum mixtum & $\mathbf{- 0 \cdot 5 9 0}$ & $\mathbf{0 \cdot 6 4 9}$ \\
Heligmosomoides glareoli & $\mathbf{0 \cdot 6 6 4}$ & $\mathbf{0 \cdot 5 1 4}$ \\
Syphacia petrusewiczi & $-0 \cdot 107$ & $0 \cdot 101$ \\
Aspiculuris tetraptera & $\mathbf{0 \cdot 2 6 8}$ & $-0 \cdot 089$ \\
Mastophorus muris & $0 \cdot 012$ & $\mathbf{- 0 \cdot 1 8 6}$ \\
Aonchotheca annulosa & $-0 \cdot 054$ & $0 \cdot 124$ \\
Trichuris sp. & $0 \cdot 070$ & $0 \cdot 076$ \\
Catenotaenia henttoneni & $\mathbf{- 0 \cdot 2 0 8}$ & $\mathbf{- 0 \cdot 2 6 4}$ \\
Paranoplocephala omphalodes & $-0 \cdot 062$ & $0 \cdot 001$ \\
Mesocestoides lineatus & $-0 \cdot 038$ & $-0 \cdot 005$ \\
Taenia mustelae & $0 \cdot 022$ & $-0 \cdot 052$ \\
Taenia martis & $-0 \cdot 073$ & $-\mathbf{0 \cdot 1 6 6}$ \\
Cladotaenia globifera & $-0 \cdot 004$ & $-0 \cdot 081$ \\
\hline \hline
\end{tabular}

* Standardized abundance data were fitted for each species. The values of those making a marked contribution to functions are emphasized in bold.

\section{Source of variation in abundance data}

Table 2 summarizes for each statistical model the percentage of variation (deviance in $\mathrm{R}$ ) explained by each of the significant terms. It is clear from these data that the major source of variation, for the 5 species that were examined quantitatively, arose from the site effect. In 4 of these 5 species there was also a significant time effect but this accounted for a considerably smaller percentage of explained deviance. The exception was $S$. petrusewicz $i$ where the year effect was much stronger, as was the interaction between site and year, with a resultant change in the relative ranking of sites between the two surveys. Intrinsic factors generally played a lesser role in explaining variation in parasite burdens.

\section{Canonical discriminant function analysis}

Canonical discriminant function (CDF) analysis revealed 5 axes that cumulatively accounted for all of the discrimination. Axis 1 (Eigenvalue =1.94) accounted for $66 \cdot 6 \%$ and Axis 2 (Eigenvalue $=0.524$ ) accounted for a further $18 \%$ of the discrimination, and therefore we did not examine Axes 3-5. Axis 1 (Table 6 and Fig. 7) contrasts H. glareoli and A. tetraptera with $H$. mixtum and C. henttoneni, and can be interpreted as reflecting differences between sites. Both $H$. glareoli and A. tetraptera were most abundant at Pilchy, and least at Urwitałt. Both $H$. mixtum and $C$. henttoneni were most abundant at Urwitalt and least at Pilchy. Axis 2 contrasts $C$. henttoneni, M. muris and T. martis against $H$. glareoli and H. mixtum. As can be seen from Table 3 the abundance of the former three all increased between 1999 and 2002 in all the sites where they occurred, whereas that of H. mixtum and 
Canonical Discriminant Functions
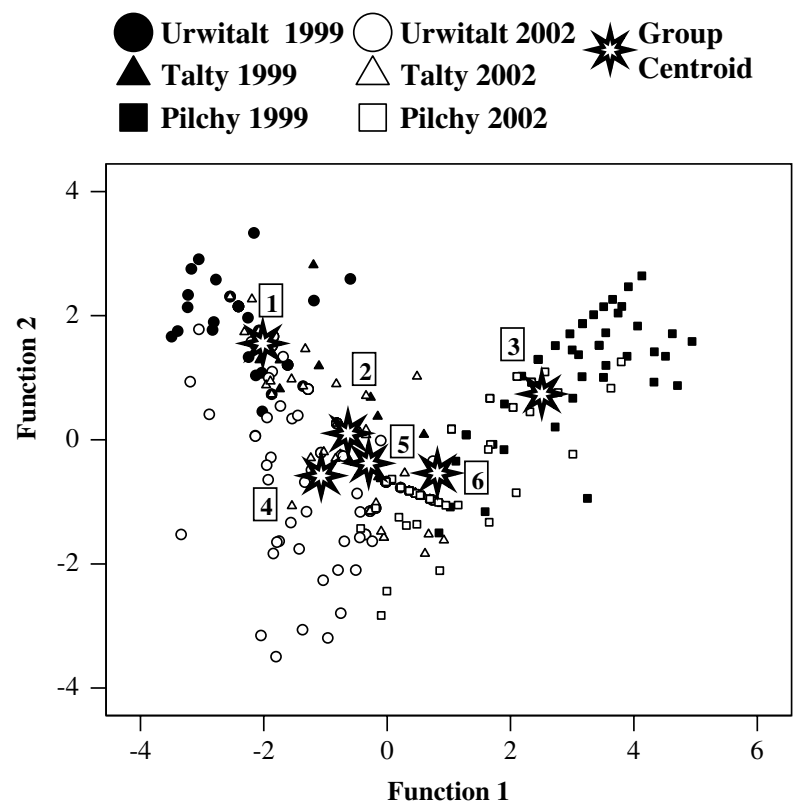

Fig. 7. Scatter plot of functions 1 and 2 derived from Canonical Discriminant Function Analysis for all 13 species of helminths in voles grouped by site and year. The correlation between each species and functions 1 and 2 is given in Table 6 . The sites are represented by different symbols, with filled-in symbols representing data from 1999 and open symbols data from 2002. The group centroids are illustrated by the stars and distinguished by the nearest number. Urwitalt is identified by 1 and 4, Talty by 2 and 5 and Pilchy by 3 and 6. Numbers 1, 2 and 3 are data from 1999 and 4, 5 and 6 from 2002.

H. glareoli declined. Therefore, this axis can be regarded as a reflection of the major changes with time, i.e. between the surveys. It is clear from the distribution of symbols and the centroids for each of the 6 subsets of data ( 3 sites and 2 years) that, to a large extent, the site effect was maintained between 1999 and 2002 (compare sequence of centroids labelled 1, 2 and 3, with the sequence 4, 5 and 6). In terms of the year effect, the centroids of all three sites have moved downwards (more negative) on axis 2 , with the greatest change at Urwitalt (centroids 1 and 4 ) and the least at Tałty (centroids 2 and 5). Overall therefore the relative order of the site effect (from left to right in Fig. 7) in both years was the same, although the difference between sites was smaller in 2002 (a slight contraction towards the centre) than in 1999.

\section{DISCUSSION}

The key objective of this work was to test for relative constancy of the infracommunity through time, in the face of spatial differences among sites: this is exactly what we found. In marked contrast to measures of regional helminth fauna and component community structure which were fluid, changing from year to year and altering in rank order between sites (see accompanying paper, Behnke et al. 2008), infracommunity structure was clearly dominated by the site effect when examined at a variety of levels (e.g. prevalence, aggregation, abundance etc.) for individual parasite species that were common and which could be treated quantitatively. In general, relevant measures showed the same rank order in 1999 and 2002 across sites. When all the abundance data were combined, standardized and analysed by Canonical Discriminant Function Analysis, year and site effects were clearly reflected in the two major axes that collectively explained $84 \%$ of the discrimination. Site was relatively more important in explaining $66 \%$ of total discrimination and remained relatively stable with time, with year of study explaining only $18 \%$. This finding is consistent with earlier studies, which have generally shown that the greatest source of variation in quantitative data (abundance) fitted to statistical models derives from differences among sites (Montgomery and Montgomery, 1989, 1990; Eira et al. 2006) rather than to changes in time (Kisielewska, 1970a; Keymer and Dobson, 1987; Bajer et al. 2005; Bugmyrin et al. 2005). Therefore, at the level of infracommunity structure, there seems to be considerable medium-term stability in these communities and, as in other helminth and protozoan infections, the site from which animals are taken is critically important (Mollhagan, 1978; Thul et al. 1985 ; Calvete et al. 2004; Booth, 2006).

The haplodiploid oxyurid nematodes can be singled out as the most problematic in studies of helminth communities and this is really because the tools we have to analyse communities cannot deal with their highly aggregated distributions. Helminth parasites are known to show aggregated distributions among hosts (Anderson and May, 1978; Haukisalmi, 1986; Shaw and Dobson, 1995). However, in the Oxyuridae in general, and in Syphacia spp. in particular, aggregation can be dramatic. In the case of $S$. petrusewiczi in this study, the overdispersion was extreme (see also Kisielewska, 1970b): so extreme that models based on negative binomial error structures would not converge satisfactorily. Not only were infections aggregated in relatively few voles, but the abundance and prevalence of $S$. petrusewicz $i$ varied more in respect of year than of site, distinguishing this species from all the others for which it was possible to analyse quantitative data. Because worm burdens could be so high, S. petrusewiczi had a disproportional influence on the derived statistics to which it contributed, particularly those concerned with regional helminth fauna and component communities (see accompanying paper, Behnke et al. 2008). The influence of this species on infracommunity statistics was far less substantial and essentially confined to analysis of total helminth 
burdens. Two other relatively uncommon, highly aggregated species were the larval stages of the cestodes $M$. lineatus, and $C$. globifera (see Behnke et al. 2008). Neither contributed significantly to infracommunity structure because their prevalence was so low.

A key finding at infracommunity level that remained stable between the two surveys was the total absence of $H$. mixtum from Pilchy and the presence instead of more intense infections with $H$. glareoli, relative to the other two sites. A third survey (2006) has confirmed this finding (Behnke et al. unpublished data). Although the prevalence and abundance of H. mixtum were lower in 2002 in both Urwitalt and Talty, the relative ranking remained the same as in 1999, with infections being more abundant and prevalent at Urwitałt in both years. Similarly, although the prevalence of $H$. glareoli fell between 1999 and 2002, it remained relatively high at Pilchy in both years. Here also, quantitative analysis revealed that the site effect explained the greater proportion of deviance in the data, and although significant, the year effect was relatively minor in comparison.

A similar pattern was shown by $M$. muris. This species was absent from a different site, Talty in 1999, and only one worm was collected here in 2002. It increased marginally at Pilchy but substantially, over 3-fold, at Urwitalt, and the figures for 2002 also contrast strongly with data collected in 1998-2000 that reported a very low prevalence at Urwitalt for this species (Bajer et al. 2005). Not surprisingly, the site effect was most important and the betweenyear effect was considerably weaker. Interestingly, M. muris was a species that was clearly subject to some degree of regulation by host sex, and unusually the bias was in the direction of female hosts. The related spirurid nematode Protospirura muricola, which also lives in the stomach of its host Acomys dimidiatus, showed a similar, but non-significant bias in favour of female mice (Behnke et al. 2004). Our finding of a female host bias for $M$. muris are supported by the data of Haukisalmi and Henttonen (2000) who also found the prevalence of $M$. muris higher in female bank voles in August and September (the same months as our surveys) in Finland. The basis of the dichotomy between the sexes in our study is not known, but may be hormonally/immunologically driven (Haukisalmi and Henttonen, 2000). However, Kisielewska $(1970 b)$ reported a male bias for this species in bank voles from various localities in Poland with 'excessive infection', but not those with 'standard' infections. In the Nearctic, Bangs (1985) also reported a male bias for this species in Myodes rutilus whilst Torres et al. (2001) found no sex bias in Eurasian badgers (Meles meles). In fact, little is known in general about the host-parasite relationship of $M$. muris, apart from reports of its occurrence and those describing its life cycle (Quentin, 1970;
Campos and Vargas, 1977, 1978). The higher prevalence and abundance in female animals is unusual for helminths, since where host sexes differ in susceptibility and resistance, it is more usual to find both prevalence and abundance higher among males, which generally show weaker immune responses to infection and being more vagile are more likely to be exposed to infective stages of parasites (Alexander and Stimson, 1988; Poulin, 1996; Zuk and McKean, 1996; Roberts et al. 1996). One possibility is that female bank voles in our study fed more often on the insect intermediate hosts of this species (Dyer and Olsen, 1967; Quentin, 1970; Campos and Vargas, 1977) and hence were more exposed to the infection, rather than being less resistant to $M$. muris. Even fleas may be intermediate hosts (Beaucournu and Chabaud, 1963; Miyata, 1939; Dyer and Olsen, 1967), although Shogaki et al. (1972) failed to find any evidence of this among the fleas they examined. If the vole fleas can act as an intermediate host infection presumably takes place during the microphagous larval stage, which is likely to be aggregated in vole nests. Pockets of local transmission in and around nests may also be mediated by the other ground insects known to act as hosts for this species. Although males generally carry more fleas (Bajer, unpublished observations), they spend less time in nests, since they have larger territories to defend in the breeding season (Gipps, 1985; Ylonen and Viitala, 1991). Clearly $M$. muris accumulates with host age, a robust finding that was evident in both years of our study through the highly significant effects of age and sex on both prevalence and abundance. This is a reflection of its slow rate of maturation with a 4-6 week pre-patent period (Dyer and Olsen, 1967; Quentin, 1970) and probable longevity, as in other closely related spirurids (Quentin, 1969; Lowrie, 2003), and is consistent with the idea that mature, rather than juvenile, females are especially at risk of infection, possibly whilst nursing young.

Another species that retained its relative ranking between the three sites was A. tetraptera. In both surveys prevalence and abundance were highest at Pilchy, intermediate at Tałty and lowest at Urwitałt, but despite this apparent stability, there were interesting changes between the surveys. Thus prevalence increased in 2002 at both Urwitałt and Tałty but remained high and constant at Pilchy, whilst abundance dropped at Pilchy and Tałty, and increased at Urwitalt. In this case therefore, although the differences between sites retained their relative ranking, the magnitude and direction of changes with time were not consistent. The increased prevalence combined with reduced abundance is unusual, since these two measures (abundance and prevalence) are more often positively correlated (Shaw and Dobson, 1995 ; Morand and Guegan, 2000). In this case the reduced abundance at two sites in 2002 is attributable primarily to fewer high-intensity infections and hence 
lower aggregation in 2002 compared with 1999. In a wider context, since the mid 1990s this nematode has shown a long-term trend of increasing prevalence and abundance from year to year in the bank vole populations, that has not yet peaked (Bajer et al. unpublished observations).

$C$. henttoneni, the only cestode to occur frequently enough to be amenable to quantitative analysis, was more often encountered at Urwitalt in both years and least so at Pilchy. Again, the relative ranking of sites was identical in both years of the survey, although there was an increase in prevalence and abundance in 2002 at all three sites. Clearly, the site effect was the most important factor influencing $C$. henttoneni.

In previous studies (Behnke et al. 2001) and in other rodents (Montgomery and Montgomery, 1989; Behnke et al. 1999, 2001, 2004), mean species richness and Brillouin's ID have both increased significantly with host age, and for both measures age explained most of the deviance in statistical models (Montgomery and Montgomery, 1989). Species richness and diversity increase with age because with increasing time after birth there is an increasing cumulative probability for exposure to parasite transmission stages, and, as long as the parasites are long-lived and do not stimulate host protective immunity, their burdens should increase with age (Hudson et al. 2006). Not surprisingly, therefore, and in common with several earlier studies, the agedependence of mean species richness and Brillouin's ID were both robust findings (Montgomery and Montgomery, 1989; Behnke et al. 2001; Bajer et al. 2005).

Averaged across all three sites, neither species richness nor Brillouin's ID changed with time (see also Bajer et al. 2005). Nevertheless, the overall means disguised the fact that there were timedependent changes in all sites, but in opposite directions. The rankings of both indices changed completely between the two years (the significant interactions between site and year). Given that most of the common species discussed above maintained their relative ranking across the three sites, and that exclusion of the rare species made no difference at all to the ranking, this is perhaps at first surprising. However, this is clearly the consequence of differences between the sites in the degree to which the prevalence and abundance of the individual common species changed with time. At Urwitalt, the number of recorded helminth species increased from 9 to 12 , some of which affected sizeable proportions of the population (e.g. $12 \%$ prevalence for T. martis), and 3 of the common species showed higher prevalence in 2002 (A. tetraptera, M. muris and C. henttoneni). Cumulatively these changes were sufficient to make species diversity and Brillouin's ID highest at Urwitalt in 2002. In contrast Pilchy, which had the highest mean species richness and Brillouin's ID in 1999 , had the lowest in 2002. The range of species recorded fell by only 1 but the greatest impact on both measures was from the $51 \%$ decline in the prevalence of $H$. glareoli by 2002 . The prevalence of all other species changed only by less than $5 \%$.

As discussed earlier (Bajer et al. 2005), the key players in determining infracommunity structure are the common species (often referred to controversially as core species, see Bush et al. 1997). While quantified measures for these species vary moderately from year to year, they nevertheless maintain a relatively stable infracommunity structure in the medium term. In the present study this was reflected in the magnitude of the site effect relative to other factors, and the maintenance of the relative ranking of sites across the two surveys for all of these species. In fact even when there was a significant interaction between site and year (e.g. H. mixtum and M. muris), it reflected either an increased divergence (M. muris) or a reduced difference (H. mixtum) between sites, not a change in relative ranking. The rarer species in the study contributed little to mean species richness or Brillouin's ID because of their low prevalence, other than on the infrequent occasions when prevalence was exceptionally higher (e.g. T. martis at Urwitalt). The contribution of these species could not be analysed quantitatively because infections were so infrequent, hence, in contrast to their contribution to component community structure where they do exert a more important influence on derived summary statistics, their contribution to infracommunity structure was negligible (see Behnke et al. 2008 for discussion of these species).

In conclusion, this study has shown that the helminth infracommunities of bank voles have a rather stable core of species that does not vary much from year to year. The exact location from which voles were sampled was critical, since most of the measures of infracommunity structure varied between sites, even when these were in relative proximity (10-25 km in our study; Montgomery and Montgomery, 1990). Over the medium term, as represented by a 3-year interval between surveys, most of the indicators of infracommunity structure relating to common species showed the same relative ranking across sites, suggesting a substantial element of predictability and stability, with the key exception of $S$. petrusewiczi. The picture was more dynamic also at the level of the derived data for infracommunities (mean species richness and Brillouin's index of diversity) and primarily because of the combination of relatively small but contrasting changes in prevalence and abundance of the common, rather than rare, species between the years of the study. It will be interesting to re-assess these sites again in the future, and to identify any longer term influences on the measures quantified here, especially in the context of changing climatic conditions which are causing concern with respect to the spread of existing parasitic infections and emerging infectious 
diseases (Brooks and Hoberg, 2007). As elsewhere, N.E. Poland is experiencing less severe winters during which reduced snow cover may impinge upon vole survival and population density, and therefore upon their helminth communities (Haukisalmi and Henttonen, 1990; Korslund and Steen, 2006; Hudson et al. 2006). Continued long-term monitoring of helminth communities in wild rodents with respect to changing climatic conditions (Haukisalmi et al. 1988; Hudson et al. 2006) will yield long-term trends in parasitic infections which will allow assessment of overall health risks from other important pathogens in the region, including those of both domestic livestock and human communities living in the region (Guernier et al. 2004; Brooks and Hoberg, 2007).

We are grateful to the M. B. Grabowski Fund for the provision of a grant for this project and the State Committee for Scientific Research (KBN), Poland and the British Council UK (BC) for additional financial support (KBN-BC Grants UM 855 and UM 930). We thank Professor M.Kozakiewicz and Dr Grzegorz Gorecki for use of the field station at Urwitałt (Department of Ecology, Institute of Zoology). We are indebted to D. Bray, T. Race, J. Dinmore, K. Frake and J. Osmond who contributed to both field and laboratory work conducted in 1999. We also thank Dr Tom Reader for his advice with statistical analyses. Finally we wish to acknowledge the support of the forestry departments responsible for the woodland sites utilized in our study (Nadlesnictwa Giżycko, Mikołajki and Orzysz).

\section{REFERENCES}

Abu-Madi, M. A., Behnke, J. M., Lewis, J. W. and Gilbert, F. S. (2000). Seasonal and site specific variation in the component community structure of intestinal helminths in Apodemus sylvaticus from three contrasting habitats in south-east England. Fournal of Helminthology 74, 7-16.

Alexander, J. and Stimson, W. H. (1988). Sex hormones and the course of parasitic infections. Parasitology Today 4, 189-193.

Anderson, R. M. and May, R. M. (1978). Regulation and stability of host-parasite population interactions. I. Regulatory processes. Fournal of Animal Ecology 47, 219-247.

Bajer, A., Behnke, J. M., Pawełczyk, A., Kuliś, K., Sereda, M. J. and Siński, E. (2005). Medium-term temporal stability of the helminth component community structure in bank voles (Clethrionomys glareolus) from the Mazury Lake District region of Poland. Parasitology 130, 213-228.

Bangs, E. E. (1985). Occurrence of the nematode Protospirura muris in Alaskan Northern red-backed voles, Clethrionomys rutilus. Canadian Field Naturalist 99, 386-388.

Beaucournu, J. C. and Chabaud, A. G. (1963). Infestation spontanée de Puces par le spiruride Mastophorus muris (Gmelin). Annales de Parasitologie (Paris) 38, 931-934.

Behnke, J. M., Bajer, A., Harris, P. D., Newington, L., Pidgeon, E., Rowlands, G., Sheriff, C.,
Kuliś-Malkowska, K., Siński, E., Gilbert, F. S. and Barnard, C. J. (2008). Temporal and between-site variation in helminth communities of bank voles (Myodes glareolus) from N.E. Poland. 1. Regional fauna and component community levels. Parasitology 135.

Behnke, J. M., Barnard, C. J., Bajer, A., Bray, D., Dinmore, J., Frake, K., Osmond, J., Race, T. and Siński, E. (2001). Variation in the helminth community structure in bank voles (Clethrionomys glareolus) from three comparable localities in the Mazury Lake District region of Poland. Parasitology 123, 401-414.

Behnke, J. M., Harris, P. D., Bajer, A., Barnard, C. J., Sherif, N., Cliffe, L., Hurst, J., Lamb, M., Rhodes, A., James, M., Clifford, S., Gilbert, F. S. and Zalat, S. (2004). Variation in the helminth community structure in spiny mice (Acomys dimidiatus) from four montane wadis in the St. Katherine region of the Sinai Peninsula in Egypt. Parasitology 129, 379-398.

Behnke, J. M., Lewis, J. W., Mohd Zain, S. N. and Gilbert, F. S. (1999). Helminth infections in Apodemus sylvaticus in southern England: interactive effects of host-age, sex and year on prevalence and abundance of infections. Fournal of Helminthology 73, 31-44.

Booth, M. (2006). The role of residential location in apparent helminth and malaria associations. Trends in Parasitology 22, 359-362.

Brooks, D. R. and Hoberg, E. P. (2007). How will global climate change affect parasite-host assemblages? Trends in Parasitology 23, 571-574.

Bugmyrin, S. V., Ieshko, E. P., Anikanova, V. A. and Bespyatova, L. A. (2005). Patterns of host-parasite interactions between the nematode Heligmosomum mixtum (Schulz, 1952) and the bank vole (Clethrionomys glareolus Schreber, 1780). Parasitologia 39, 414-422.

Bush, A. O., Lafferty, K. D., Lotz, J. M. and Shostak, A. W. (1997). Parasitology meets ecology on its own terms: Margolis et al. revisited. Fournal of Parasitology 83, 575-583.

Calvete, C., Blanco-Aguiar, J. A., Virgós, E., CabezasDiaz, S. and Villafuerte, R. (2004). Spatial variation in helminth community structure in the red-legged partridge (Alectoris rufa L.): effects of definitive host density. Parasitology 129, 101-113.

Campos, M. Q. and Vargas, M. V. (1977). Biologia de Protospirura muricola Gedoelst, 1916 y Mastophorus muris (Gmelin, 1790) (Nematoda: Spiruridae), en Costa Rica. I. Huespedes intermediarios. Revista de Biologia Tropical 25, 191-207.

Campos, Q. M. and Vargas, E. M. (1978). The biology of Protospirura muricola and Mastophorus muris (Nematoda: Spiruridae) in Costa Rica II. Definitive hosts. Revista de Biologia Tropica 26, 199-211.

Carleton, M. D., Musser, G. G. and Pavlinov, I. Â. (2003). Myodes Pallas, 1811, is the valid name for the genus of red-backed voles. In Systematics, Phylogeny and Paleontology of Small Mammals. Proceedings of the International Conference devoted to the 90th anniversary of Prof. I. M. Gromov, November 2003, Saint Petersburg (ed. Averianov, A. and Abramson, N.), pp. $96-98$.

Dyer, W. G. and Olsen, O. W. (1967). Biology of Mastophorus numidica (Seurat, 1914) Read and Millemann, 1953 (Nematoda: Spiruridae) with a 
description of the juvenile stages. Proceedings of the Helminthological Society of Washington 34, 98-103.

Eira, C., Torres, J., Vingada, J. and Miquel, J. (2006). Ecological aspects influencing the helminth community of the wood mouse Apodemus sylvaticus in Dunas de Mira, Portugal. Acta Parasitologica 51, 300-308.

Elliott, J. M. (1977). Some Methods for the Statistical Analysis of Samples of Benthic Invertebrates. Freshwater Biological Association, Cumbria, UK.

Ferrari, N., Cattadori, I. M., Nespereira, J., Rizzoli, A. and Hudson, P. J. (2004). The role of host sex in parasite dynamics: field experiments on the yellownecked mouse Apodemus flavicollis. Ecology Letters 7, 88-94.

Gipps, J. H. W. (1985). The behaviour of bank voles. In The Ecology of Woodland Rodents Bank Voles and Wood Mice. Symposia of the Zoological Society of London, 55, 61-87. The Zoological Society of London, Clarendon Press, Oxford, UK.

Gregory, R. D., Montgomery, S. S. J. and Montgomery, W. I. (1992). Population biology of Heligmosomoides polygyrus (Nematoda) in the wood mouse. Fournal of Animal Ecology 61, 749-757.

Guernier, V., Hochberg, M. E. and Guegan, J.-F. (2004). Ecology drives the worldwide distribution of human diseases. PLoS Biology 2, 740-746.

Haukisalmi, V. (1986). Frequency distributions of helminths in microtine rodents in Finnish Lapland. Annales Zoologici Fennici 23, 141-150.

Haukisalmi, V. and Henttonen, H. (1990). The impact of climatic factors and host density on the long-term population dynamics of vole helminths. Oecologia $\mathbf{8 3}$, 309-315.

Haukisalmi, V. and Henttonen, H. (2000). Variability of helminth assemblages and populations in the bank vole Clethrionomys glareolus. Polish Fournal of Ecology 48 (Suppl.) 219-231.

Haukisalmi, V., Henttonen, H. and Tenora, F. (1987). Parasitism by helminths in the grey-sided vole (Clethrionomys rufocanus) in northern Finland: influence of density, habitat and sex of the host. Fournal of Wildlife Diseases 23, 233-241.

Haukisalmi, V., Henttonen, H. and Tenora, F. (1988). Population dynamics of common and rare helminths in cyclic vole populations. Fournal of Animal Ecology 57, 807-825.

Hudson, P. J., Cattadori, I. M., Boag, B. and Dobson, A. P. (2006). Climate disruption and parasite-host dynamics: patterns and processes associated with warming and the frequency of extreme climatic events. Fournal of Helminthology 80, 175-182.

Janovy, J. Jr., Clopton, R. E., Clopton, D. A., Snyder, S. D., Efting, A. and Krebs, L. (1995). Species density distributions as null models for ecologically significant interactions of parasite species in an assemblage. Ecological Modelling 77, 189-196.

Kennedy, C. R., Bush, A. O. and Aho, J. M. (1986). Patterns in helminth communities: why are birds and fish different? Parasitology 93, 205-215.

Kennedy, C. R. and Hartvigsen, R. A. (2000). Richness and diversity of intestinal metazoan communities in brown trout Salmo trutta compared to those of eels Anguilla anguilla in their European heartlands. Parasitology 121, 55-64.
Keymer, A. E. and Dobson, A. P. (1987). The ecology of helminths in populations of small mammals. Mammal Review 17, 105-116.

Kisielewska, K. (1970a). Ecological organization of intestinal helminth groupings in Clethrionomys glareolus (Schreb.) (Rodentia). 1. Structure and seasonal dynamics of helminth groupings in a host population in the Bialowieża National Park. Acta Parasitologica Polonica 18, 121-147.

Kisielewska, K. (1970b). Ecological organization of intestinal helminth groupings in Clethrionomys glareolus (Schreb.) (Rodentia). IV. Spatial structure of a helminth grouping within the host population. Acta Parasitologica Polonica 18, 177-196.

Kisielewska, K. (1971). Intestinal helminths as indicators of the age structure of Microtus arvalis Pallas, 1778 population. Bulletin de L'Academie Polonaise des Sciences. Serie des Sciences Biologiques Cl.II, 19, 275-282.

Korslund, L. and Steen, H. (2006). Small rodent winter survival: snow conditions limit access to food resources. Fournal of Animal Ecology 75, 156-166.

Langley, R. and Fairley, J. S. (1982). Seasonal variations in infestations of parasites in a wood mouse Apodemus sylvaticus population in the west of Ireland. Fournal of Zoology, London 198, 249-261.

Lewis, J. W. (1968). Studies on the helminth parasites of voles and shrews from Wales. Fournal of Zoology, London 154, 313-331.

Lowrie, F. M. (2003). Host-Parasite Relationship of Protospirura muricola, a Spirurid Nematode. M.Phil thesis, University of Nottingham, UK.

Margolis, L., Esch, G. W., Holmes, J. C., Kuris, A. M. and Schad, G. A. (1982). The use of ecological terms in parasitology (report of an ad hoc committee of The American Society of Parasitologists). Fournal of Parasitology 68, 131-133.

Miyata, J. (1939). Studies on the life history of the nematode Protospirura muris (Gmelin) parasitic in the stomach of the rat, especially on the relation of the intermediate hosts, cockroaches, skin moth and rat fleas. Volumen jubilare pro Professore Sadao Yoshida, Osaka, Japan 1, 101-136 (In Japanese.)

Mollhagan, T. (1978). Habitat influence on helminth parasitism of the cotton rat in western Texas, with remarks on some of the parasites. The Southwestern Naturalist 23, 401-407.

Montgomery, S. S. J. and Montgomery, W. I. (1989). Spatial and temporal variation in the infracommunity structure of helminths of Apodemus sylvaticus (Rodentia: Muridae). Parasitology 98, 145-150.

Montgomery, S. S. J. and Montgomery, W. I. (1990). Structure, stability and species interactions in helminth communities of wood mice Apodemus sylvaticus. International Fournal for Parasitology 20, 225-242.

Morand, S. and Guegan, J. F. (2000). Distribution and abundance of parasite nematodes: ecological specialization, phylogenetic constraints or simply epidemiology? Oikos 88, 563-573.

Poulin, R. (1993). The disparity between observed and uniform distributions: a new look at parasite aggregation. International Fournal for Parasitology 23, 937-944. 
Poulin, R. (1996). Sexual inequalities in helminth infections: a cost of being a male? The American Naturalist 147, 287-295.

Poulin, R. (1997). Species richness of parasite assemblages: evolution and patterns. Annual Reviews in Ecology and Systematics 28, 341-358.

Quentin, J. C. (1969). Cycle biologique de Protospirura muricola Gedoelst 1916 (Nematoda; Spiruridae). Annales de Parasitologie (Paris) 44, 485-504.

Quentin, J. C. (1970). Morphogenese larvaire du spiruride Mastophorus muris (Gmelin, 1790). Annales de Parasitologie (Paris) 45, 839-855.

Roberts, C. W., Satoskar, A. and Alexander, J. (1996). Sex steroids, pregnancy-associated hormones and immunity to parasitic infection. Parasitology Today 12, 382-388.

Rohlf, F. J. and Sokal, R. R. (1995). Statistical Tables. W. H. Freeman and Company, San Francisco.

Shaw, D. J. and Dobson, A. P. (1995). Patterns of macroparasite abundance and aggregation in wildlife populations: a quantitative review. Parasitology 111, S111-S127.

Shogaki, Y., Mizuno, S. and Itoh, H. (1972). On Protospirura muris (Gmelin) a parasic nematode of the brown rat in Nagoya City. Fapanese Fournal of Parasitology 21, 28-38.

Tenora, F. and Stanek, M. (1995). Changes of the helminthofauna in several Muridae and Arvicolidae at Lednice in Moravia. II. Ecology. Acta Universitatis
Agriculturae et Silviculturae Mendelianae Brunensis 43, 57-65.

Thul, J. E., Forrester, D. J. and Abercrombie, C. L. (1985). Ecology of parasitic helminths of wood ducks, Aix sponsa, in the Atlantic flyway. Proceedings of the Helminthological Society of Washington 52, 297-310.

Torres, J., Miquel, J. and Motje, M. (2001). Helminth parasites of the Eurasian badger (Meles meles L.) in Spain: a biogeographic approach. Parasitology Research 87, 259-263.

Venables, W. N. and Ripley, B. D. (1997). Modern Applied Statistics with S-Plus. Springer, New York.

Washington, H. G. (1984). Diversity, biotic and similarity indices. A review with special relevance to aquatic ecosystems. Water Research 18, 653-694.

Wilson, D. E. and Reeder, D. M. (2005). Mammal Species of the World. A Taxonomic and Geographic Reference, 3rd Edn. Johns Hopkins University Press, Baltimore, USA.

Xu, R. (2003). Measuring explained variation in linear mixed effects models. Statistics in Medicine 22, $3527-3541$.

Ylonen, H. and Viitala, J. (1991). Social overwintering and food distribution in the bank vole Clethrionomys glareolus. Hoarctic Ecology 14, 131-137.

Zuk, M. and McKean, K. A. (1996). Sex differences in parasitic infections: patterns and processes. International Fournal for Parasitology 26, 1009-1024. 